ARTICLE

\title{
Maintenance of sarcomeric integrity in adult muscle cells crucially depends on Z-disc anchored titin
}

\author{
Sandra Swist (10 ${ }^{1 凶}$, Andreas Unger ${ }^{2}$, Yong Li², Anja Vöge ${ }^{1}$, Marion von Frieling-Salewsky², Asa Skärlén ${ }^{3}$, \\ Nicola Cacciani ${ }^{4}$, Thomas Braun (10 ${ }^{5}$, Lars Larsson (D) $^{4} \&$ Wolfgang A. Linke (i) ${ }^{2 \times}$
}

The giant protein titin is thought to be required for sarcomeric integrity in mature myocytes, but direct evidence for this hypothesis is limited. Here, we describe a mouse model in which Z-disc-anchored TTN is depleted in adult skeletal muscles. Inactivation of TTN causes sarcomere disassembly and Z-disc deformations, force impairment, myocyte de-stiffening, upregulation of TTN-binding mechanosensitive proteins and activation of protein qualitycontrol pathways, concomitant with preferential loss of thick-filament proteins. Interestingly, expression of the myosin-bound Cronos-isoform of TTN, generated from an alternative promoter not affected by the targeting strategy, does not prevent deterioration of sarcomere formation and maintenance. Finally, we demonstrate that loss of Z-disc-anchored TTN recapitulates muscle remodeling in critical illness 'myosinopathy' patients, characterized by TTN-depletion and loss of thick filaments. We conclude that full-length TTN is required to integrate Z-disc and A-band proteins into the mature sarcomere, a function that is lost when TTN expression is pathologically lowered.

\footnotetext{
${ }^{1}$ Department of Systems Physiology, Ruhr University Bochum, D-44780 Bochum, Germany. ${ }^{2}$ Institute of Physiology II, University of Munster, D-48149 Munster, Germany. ${ }^{3}$ Department of Clinical Neuroscience, Clinical Neurophysiology, Karolinska Institute, SE-171 77 Stockholm, Sweden. ${ }^{4}$ Department of Physiology and Pharmacology, Karolinska Institute, SE-171 77 Stockholm, Sweden. ${ }^{5}$ Department of Cardiac Development and Remodeling, Max Planck

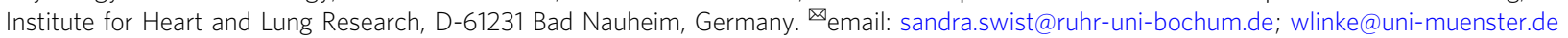


S keletal muscles are required for gait, posture, and many additional functions. As much as $80-90 \%$ of the volume of an adult skeletal muscle cell is occupied by the contractile units known as the sarcomeres. These structures are multiprotein assemblies of paracrystalline order, which essentially consist of three filament systems bordered by Z-discs: actin-based thin filaments, myosin-based thick filaments, and titin filaments. The giant titin proteins (TTN) span half-sarcomeres from the Zdisc to the M-band ${ }^{1}$ and overlap with TTN proteins from adjacent half-sarcomeres ${ }^{2}$. Several different alternative transcripts are generated by the TTN gene, including full-length isoforms (3-3.8 $\mathrm{MDa}$ ) termed $\mathrm{N} 2 \mathrm{~B}, \mathrm{~N} 2 \mathrm{BA}$ (in the heart) and N2A (in skeletal muscles), as well as the small, Z-disc-anchored Novex-3 isoform $(\sim 0.7 \mathrm{MDa})^{3,4}$. Moreover, the recently discovered Cronos isoform arises from an alternative transcription start site $>150$ kilobases (kb) downstream of the canonical promoter, in Ttn intron $239^{5}$. Ever since its discovery, TTN has been thought to hold the sarcomere together and provide it with stability and elasticity ${ }^{6,7}$. The role of TTN for the elasticity and stretch-dependent passive tension of myocytes is now well established ${ }^{8,9}$. Titin-based elastic force also complements and regulates the contractile force generated by actin and myosin ${ }^{10,11}$. Furthermore, TTN determines the length of the sarcomeric thick filaments ${ }^{12}$ and is important for de-novo sarcomere assembly ${ }^{13-17}$. It has also been frequently suggested that TTN is relevant for the maintenance of the mature sarcomere. This function has been more difficult to assess and details have remained unclear. However, TTN is necessary for cardiac development and its absence leads to early embryonic lethality ${ }^{13,14,18}$.

Here, we report an inducible, conditional TTN-knockout (KO) mouse model targeting the TTN isoforms expressed from the canonical Ttn promoter in adult skeletal muscles. Our approach differs from previous strategies, because earlier TTN-deficient rodent models either preserved the structural continuity of the protein, e.g., by deleting only the cardiac $\mathrm{N} 2 \mathrm{~B}$-element ${ }^{19}$, certain I-band immunoglobulin (Ig) domains ${ }^{20,21}$, the PEVKsegment $^{22,23}$, or the distal M-band titin ${ }^{15,16,24}$, or they were designed to partially or completely eliminate TTN at early stages of development ${ }^{14-16,18,25}$. In our model, we find that inactivation of N2A and Novex-3 TTN in adult skeletal muscles reduces muscle mass, contractile strength, and myocyte stiffness, causes sarcomeric disintegration along with Z-disc aggregation and streaming, and results in preferential loss of thick-filament proteins. TTN-depletion also causes upregulation of titin-binding proteins involved in mechanotransduction and activates components of the protein quality control (PQC) machinery, providing additional insight into the role of TTN in sarcomere maintenance and protein turnover. Strikingly, expression of Cronos is unable to prevent cardiac embryonic lethality or disassembly of adult skeletal muscle sarcomeres. Our findings prove the longhypothesized crucial function of TTN for protein homeostasis in mature muscle sarcomeres.

Interestingly, our conditional TTN KO-mouse model recapitulates key changes observed in the skeletal muscles of critically ill intensive care unit (ICU) patients with acute quadriplegic myopathy (critical illness myopathy (CIM) or myosinopathy), which is seen in up to $\sim 30 \%$ of ICU patients ${ }^{26}$. A hallmark of CIM is the preferential loss of myosin and myosin-associated proteins but not thin-filament proteins in the sarcomeres of proximal and distal skeletal muscles ${ }^{27}$. We find that CIM patient muscles show not only preferential loss of thick filaments but also suffer from reduced TTN content, while sarcomeric actin and the major thinfilament protein nebulin are largely preserved. Our results suggest that downregulation of TTN is a contributing factor in the pathogenesis of CIM, and presumably also in other types of muscle atrophy.

\section{Results}

Full-length titin is crucial for de-novo sarcomerogenesis. We initially generated titin 'knockout-first' heterozygous $\mathrm{Ttn}^{\mathrm{tm} l \mathrm{a} /+}$ mice with conditional potential, targeting the N-terminus of TTN (Supplementary Fig. 1, Supplementary Fig. 2, Supplementary Fig. 3). Heterozygous mice were healthy but no homozygous mutant mice were born, because titin-deficient embryos died around E10 (Supplementary Fig. 2). Interestingly, expression of Cronos, which was not targeted by our approach, was unaltered in mutant embryos (Supplementary Fig. 2). Therefore, Z-discintegrated TTN is required for cardiac sarcomerogenesis and Cronos does not rescue cardiac embryonic lethality.

Inducible skeletal muscle-specific $T \boldsymbol{t} n$ removal in adult mice. Next, we generated the conditional $\operatorname{Ttn}^{\mathrm{tm} 1 \mathrm{c}}$ allele by deleting the lacZ reporter and neomycin selection cassettes using FLP recombinase, preventing embryonic lethality of the $\operatorname{Ttn}^{\text {tmla }}$ mice (Fig. 1a, b). Homozygous $\mathrm{Ttn}^{\mathrm{tm} 1 \mathrm{c} / \mathrm{tm} 1 \mathrm{c}}$ animals were fertile and had no obvious phenotype. Breeding of $\mathrm{Ttn}^{\mathrm{tm} 1 \mathrm{c} / \mathrm{tm} 1 \mathrm{c}}$ animals with ACTA1-rtTA;tetO-cre mice ${ }^{28}$, in which CRE-expression is driven by reverse tetracycline-controlled transactivator (rtTA) under the human alpha skeletal muscle actin 1 (ACTA1) promoter, enabled us to delete Ttn exons 4-6 in adult skeletal myocytes upon doxycycline (dox) administration (Fig. 1a). To monitor the effectiveness of dox-treatment, we studied Rosa26-LacZ/Tg (ACTA1-rtTA,tetO-cre) reporter mice, which showed strong Bgalactosidase staining in fatigue-resistant (diaphragm, soleus) and fast skeletal muscles, but not in heart, lung or liver after 30 days of treatment (Supplementary Fig. 4).

Inactivation of Ttn after 30 days of dox-treatment was verified by RT-PCR, using primers to exons 2 and 8 (Fig. 1c). Deletion of Ttn exons 4-6 induced a frameshift leading to premature stop codons, which resulted in loss of full-length Ttn mRNA by nonsense-mediated decay and loss of TTN protein (Fig. 1d). To rule out the generation of new TTN isoforms with alternative $\mathrm{N}$ termini from the mutant allele ${ }^{18}$, we performed quantitative RTPCR. Expression of Ttn exons 3-4 and 20-21 was reduced by $99 \%$ and $\sim 84 \%$, respectively, in MUT vs. WT gastrocnemius muscles (Fig. 1e). In contrast, expression of Cronos remained unaltered in MUT mice, whereas Ttn exons 239-240 flanking the Cronos promoter region showed the anticipated $\sim 90 \%$ reduction (Fig. 1e). Likewise, in situ hybridizations with a riboprobe detecting Ttn exon 20-24 transcripts clearly demonstrated loss of WT Ttn mRNA (Fig. 1f).

TTN loss causes muscle atrophy and reduces muscle strength. Profound weight loss began in Ttn ${ }^{\mathrm{tm} 1 \mathrm{c} / \mathrm{ltml} \text {; }}$ ACTA1-rtTApos;tetO-

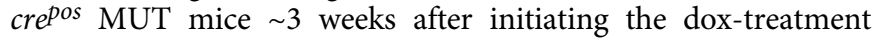
(Fig. 2a). MUT mice had $7.1 \pm 1.8 \%$ (mean \pm SD) lower body weight than WT mice ( $p=0.0026$; WT, $n=10$; MUT, $n=11$ ) on day 21 of dox-treatment, and $17 \pm 1.9 \%(p<0.001$; WT, $n=9$; MUT, $n=10$ ) lower weight on day 30 (Fig. 2a, Supplementary Fig. 5a). Individual muscles already lost weight in some mice, even when whole body weights were still unaltered (Supplementary Fig. 5b). The experiment was terminated, and tissues were collected, when animals had lost maximally $20 \%$ of their initial weight.

Dox-treated MUT mice performed significantly worse than control animals in a four-limb hanging test (4LHT) (Fig. 2b, Supplementary Fig. 5c). MUT mice showed reduced hang time after 24 days of dox-treatment $(6.8 \pm 1.5$ min (mean $\pm \mathrm{SD})$, versus $\sim 12.5$ min before treatment $(p=0.0004, n=7))$. On day 31 of treatment, the hang time was reduced to only $\sim 20 \%$ of the initial value (Fig. 2b). 
a

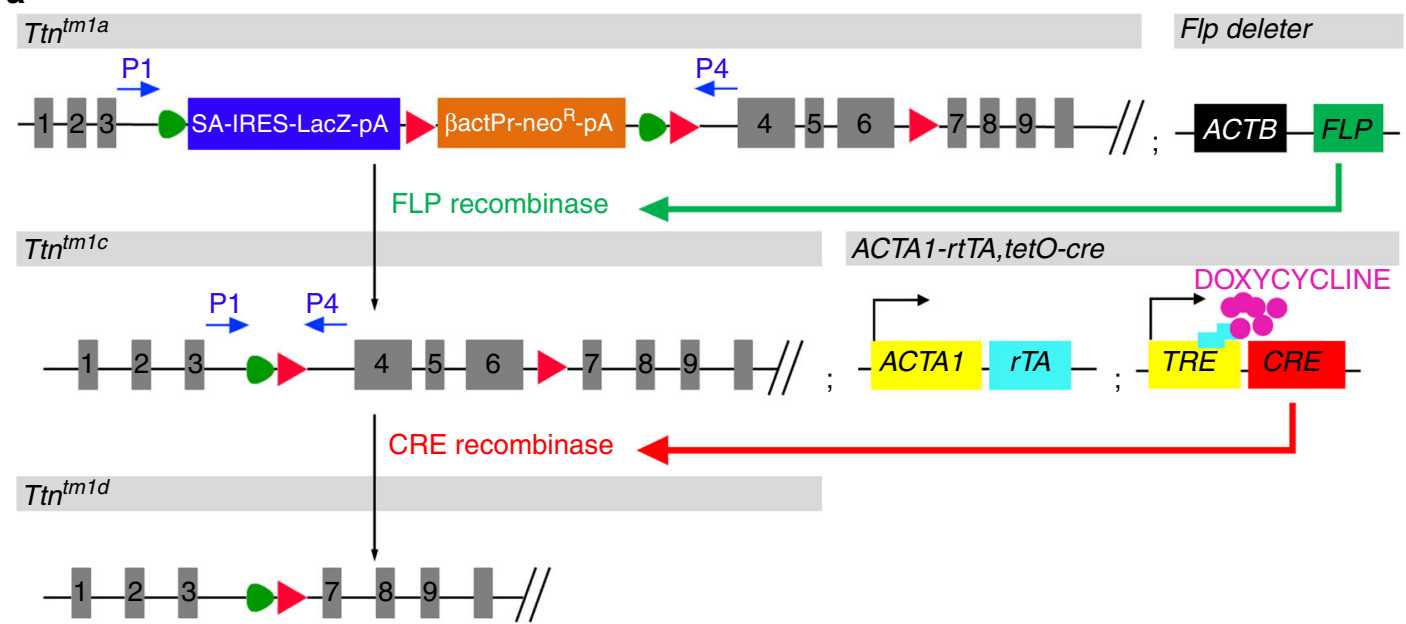

b

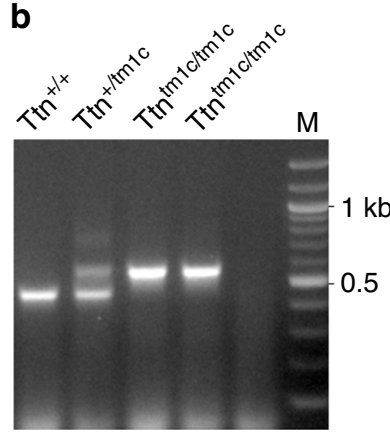

e Ttn exon 3-4

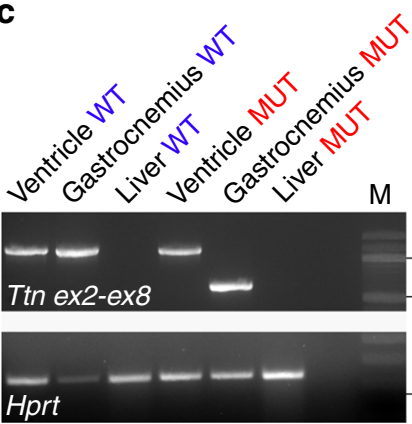

d

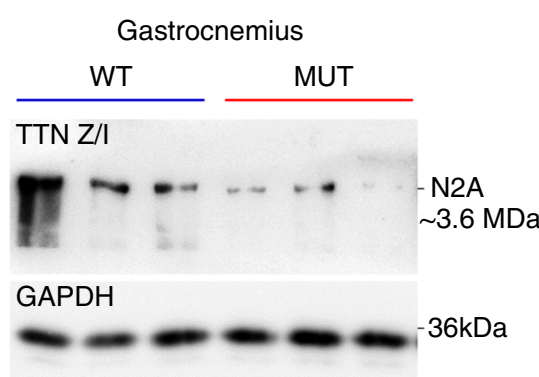

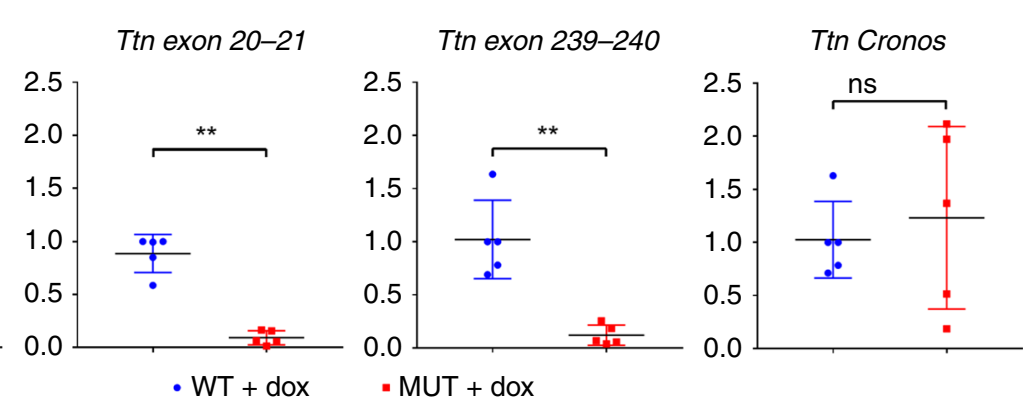

f
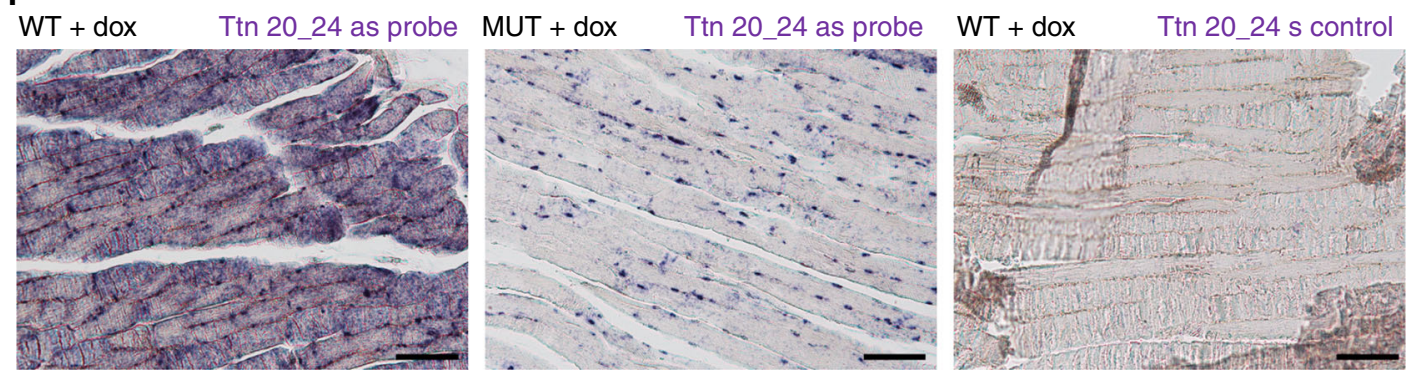

Histological analysis by hematoxylin-and-eosin staining of skeletal muscle revealed reduced myofiber diameters and some centralized nuclei in MUT (Fig. 2c), indicating muscle atrophy. Both the number of centralized nuclei (Fig. 2 d) and the mean fiber cross-sectional area (Fig. 2e) were already altered in MUT muscles after 21 days of dox-treatment.

TTN depletion disrupts the normal striated sarcomere pattern. Immunostaining against different TTN epitopes (Fig. 3a) on paraffin sections of $M$. gastrocnemius showed increased disorder and reduced signal intensities in MUT vs. WT myocytes (Fig. 3b-f). Antibodies to the TTN Z/I-junction (Z/I2080) demonstrated protein loss or reduction in many myocytes within the same muscle (Fig. 3b). The proportion of cells lacking regular Z-disc protein striations reached $\sim 20 \%$ and $\sim 40 \%$ after 21 days and $\sim 30$ days, respectively, of doxtreatment (Fig. 3c). Immunostaining with Novex-3-specific antibodies confirmed the absence of this TTN isoform in most MUT cells (Fig. 3d). Loss of TTN was always accompanied by disruption of the striated patterns of Z-disc-based a-actinin 
Fig. 1 Inducible skeletal muscle-specific titin knockout. a Mutant Ttn ${ }^{\text {tmla }}$ allele generation, with the reporter/selection cassette inserted upstream of Ttnexons 4-6. This cassette contains an FRT-site (green triangles), followed by a LacZ-trapping element (blue square) with a strong splice acceptor (SA) and internal ribosomal entry site (IRES). LoxP-sites are indicated by red triangles. The first loxP-site is followed by neomycin (neoR) under the control of the human $\beta$-actin promoter ( $\beta$ actPr), a simian virus terminator and polyadenylation signal ( $\mathrm{pA}$ ), a second FRT-site, and a second loxP-site. A third loxP-site is inserted downstream of the critical exons. The selection and lacZ cassettes were deleted by FLP recombinase-expressing mouse strain (Flp-deleter). Doxinduced CRE-recombinase expression in the hybrid line Ttn ${ }^{\mathrm{tm} 1 \mathrm{c} / \mathrm{Itmlc} ; A C T A 1-r t T A ; t e t O-c r e ~ m e d i a t e d ~ r e c o m b i n a t i o n ~ o f ~ t h e ~ r e m a i n i n g ~ l o x P-s i t e s ~ a n d ~}$ removed exons 4-6 (frameshift). b Agarose gels resolving genotyping PCR products from WT ( $\mathrm{Ttn}{ }^{+/+}$), heterozygous ( $\mathrm{tn}^{+/ t m 1 \mathrm{c}}$ ) and homozygous MUT

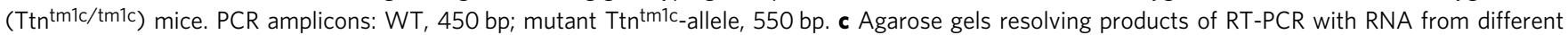
tissues of dox-treated WT and MUT Ttn ${ }^{\text {tmlc; }}$ ACTA1-rtTA;tetO-cre mice. Primers spanning exons 2-8 (1.25 kb) revealed a $0.6 \mathrm{~kb}$ amplicon in gastrocnemius but not cardiac (ventricle) muscle, demonstrating skeletal muscle-specific loss of exons 4-6. d TTN N2A-protein expression in WT and MUT gastrocnemius detected by western blot using antibodies to Z/I-band titin (2080). Loading control was GAPDH. Gels shown in b) are representative of $>5$ gels/condition, in $\mathbf{c}$ and d) 2 condition. e Quantitative RT-PCR analysis proving loss of full-length TTN RNA in dox-treated MUT vs. WT mice, using primers to exons 3-4 ( $p=0.0079)$ and exons located $3^{\prime}$ to the deletion, specifically, 20-21 $(p=0.0079)$ and 239-240 ( $\left.p=0.0079\right)$; the alternative Cronos Ttn transcript remained unaltered $(p=0.7937) . n=5$ animals/group; data are mean \pm SD. ${ }^{\star \star} p<0.01$; ns non-significant in unpaired, two-tailed Mann-Whitney $t$-test. f RNA in situ hybridizations (representative of 2-4 images/condition) with antisense (as) riboprobe targeting Ttn-exons 20-24, which detected Ttn-RNA in both cytoplasm and nuclei of WT but only in nuclei of MUT cells. Sense (s) Ttn-exons 20-24 riboprobe served as negative control. Bars, $100 \mu \mathrm{m}$. All data were obtained after $\sim 30$ days of dox-treatment. Source data are provided as a Source Data file.

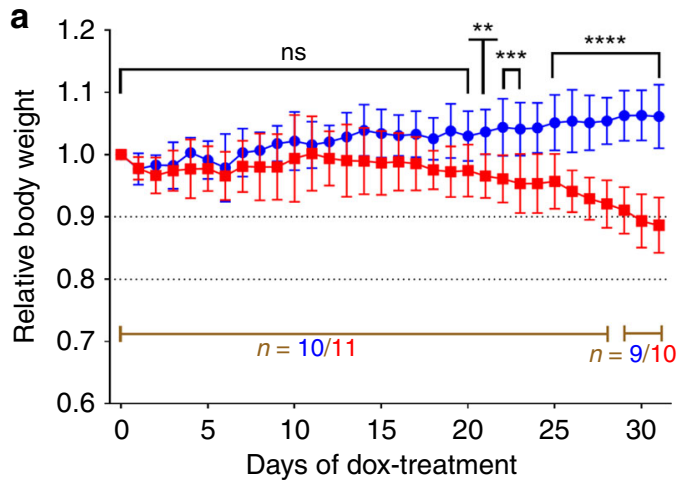

C

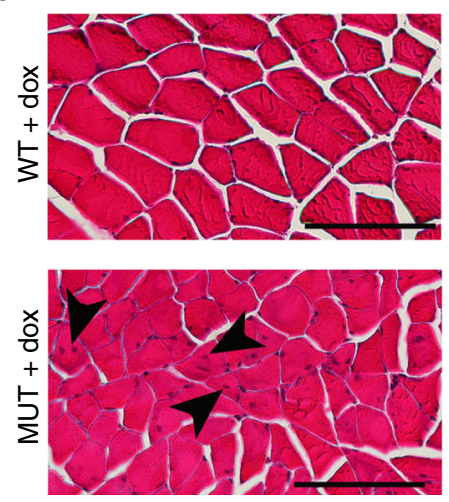

d

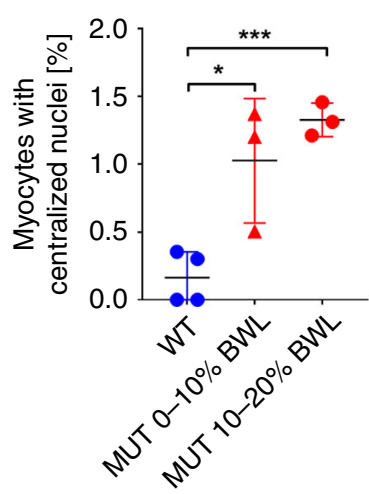

b

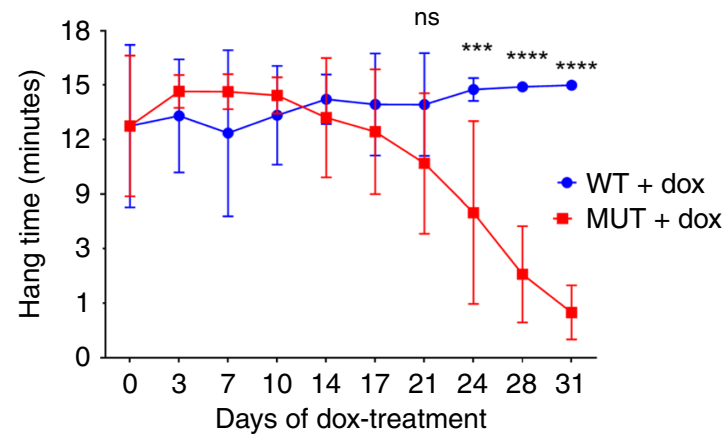

e

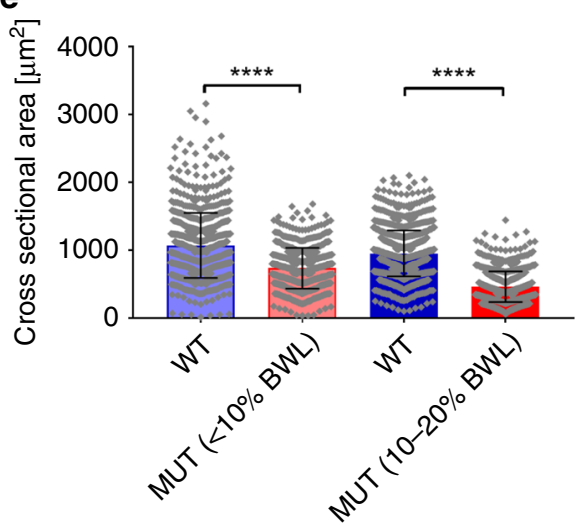

Fig. 2 Titin depletion leads to muscle remodeling and reduction in muscle strength. a Alterations in body weight with time in MUT and WT Ttn ${ }^{\text {tmic. }}$ ACTA1-rtTA;tetO-cre mice during dox-treatment. Data were normalized to the body weight immediately before dox-treatment. Beginning around day 21 , there was a significant difference between WT and MUT ( $p=0.0068$; for WT, $n=10$ animals ( 9 for the last three time points) and for MUT, $n=11$ animals (10 for the last three time points)). b Alterations in muscle strength during dox-treatment of MUT vs. WT mice measured in the 4-limb hanging test. Hang time was plotted against duration of treatment. From day 24 of dox-treatment onward, MUT mice had significantly reduced hang times $(p=0.0004 ; n=7$ animals/group). c Representative cross sections (out of 8-10 images recorded per condition) of hematoxylin and eosin stained M. triceps brachii from WT and MUT mice after 30 days of dox-treatment. Arrowheads point to centralized nuclei. Bars, $100 \mu \mathrm{m}$. $\mathbf{d}$ Percentage of myofibers presenting with centralized nuclei in dox-treated WT $(n=4)$ and MUT mice with a body weight loss (BWL) of $<10 \%(n=3)$ and $10-20 \%(n=3)$, respectively, relative to body weight before treatment. e Cross-sectional area of myofibers $(n=630)$ from WT and MUT Ttn ${ }^{\text {tmlc; }}$ ACTA1-rtTA;tetO-cre mice $(\sim 30$ days of dox-treatment); $n=3$ mice/group. Data are mean \pm SD. ${ }^{\star \star \star *} p<0.0001 ;{ }^{* \star \star} p<0.001 ;{ }^{* \star} p<0.01$, in 2-way-ANOVA, followed by Sidak's multiple comparisons test (a and $\left.\mathbf{b}\right)$; unpaired, two-tailed Student's t-test (d); or two-tailed Mann-Whitney test (e). Source data are provided as a Source Data file.

(ACTN2, Fig. 3b, e, f) and A-band-based myosin heavy chain (MyHC) (Fig. 3d). Furthermore, both the TTN-kinase domain (M-band) and the Cronos N-terminus (I/A-junction) lost their regular striated appearance in affected MUT cells (Fig. 3e, f). Thus, the highly ordered repetitive sarcomere structure depends on the presence of Z-disc-anchored TTN isoforms, whereas Cronos does not prevent deterioration of sarcomeres. 
a

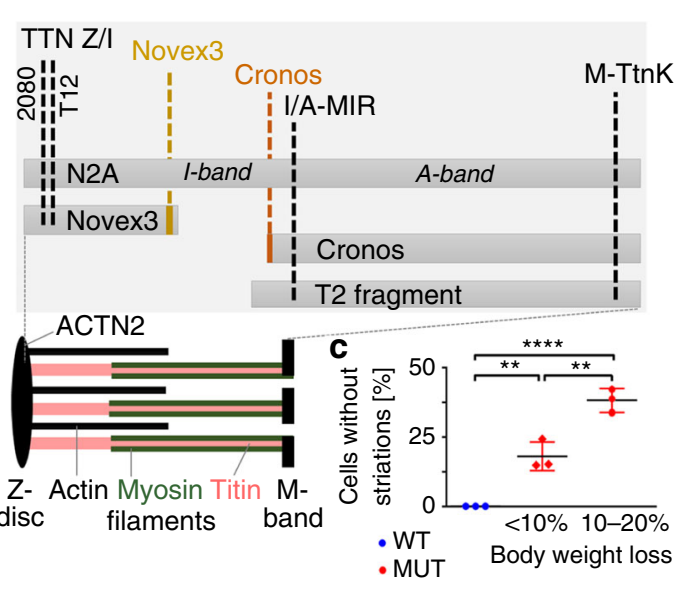

b $\operatorname{TTN}(Z / /-2080)$
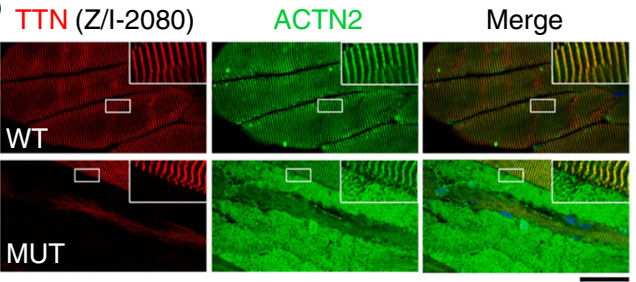

d TTN (Novex3)

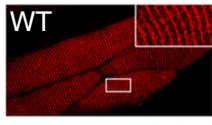

$\mathrm{MyHC}$

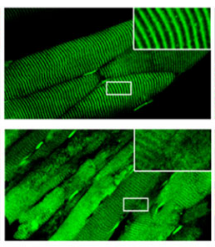

Merge

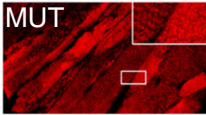

f $\operatorname{TTN}(\mathrm{M}-\mathrm{TtnK})$
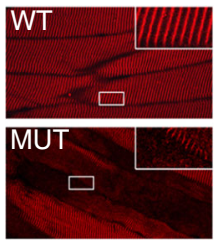

ACTN2
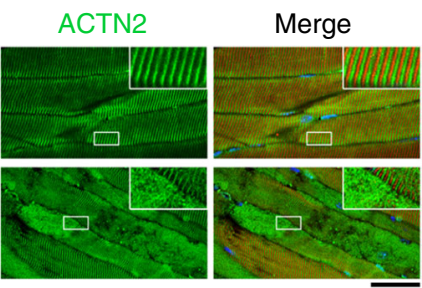

g

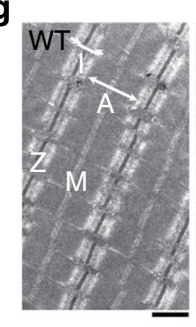

i
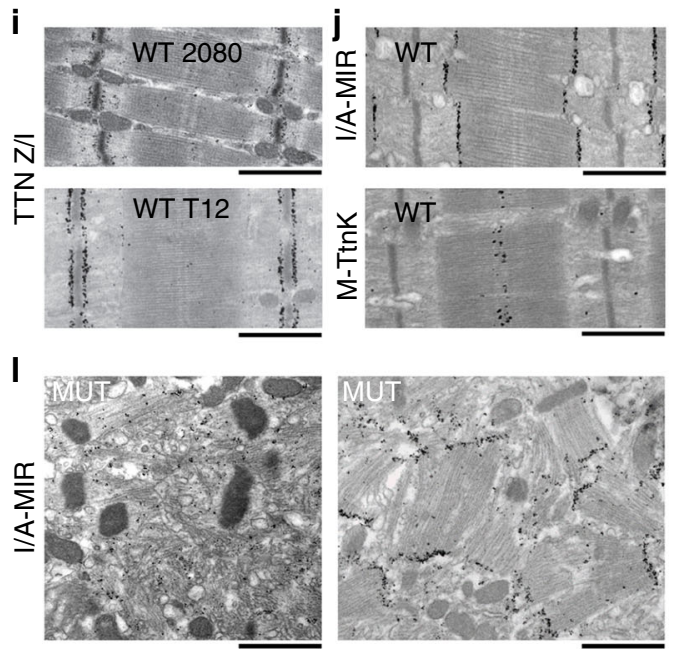

,

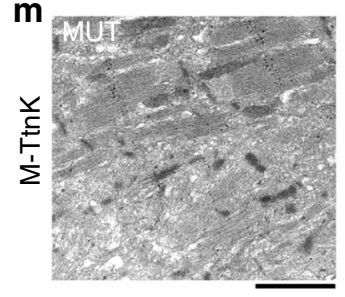

h

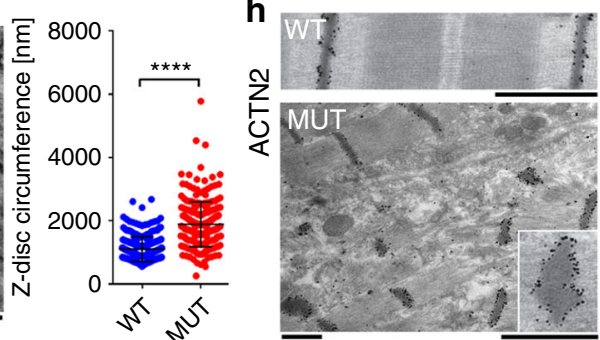

$\mathbf{k}$
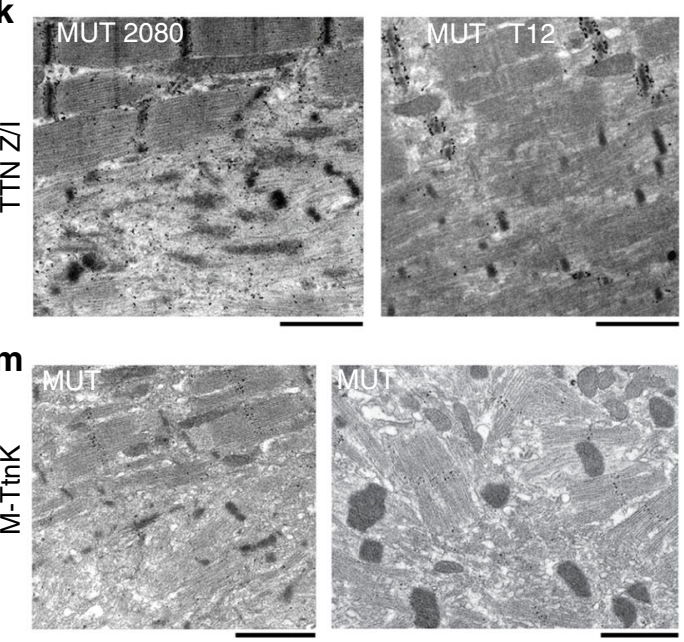

the parallel orientation of myofibrils. (3) Appearance of Z-discs as thickened rod-shaped or irregularly shaped structures with increased average circumference (Fig. 3g); Z-disc shrinking or extensive streaming were frequent. (4) Despite the continuous loss of thick filaments, A-bands were sometimes well-preserved. Moreover, we observed cells with prominent morphological aberrations in one region, while other regions still contained normal sarcomeres, and profoundly altered myocytes next to cells that still appeared healthy (Fig. 3g). Similar patterns of disruption were already present in MUT muscles after 21 days of
TTN loss disconnects A- and Z-bands and promotes aggregation. Electron micrographs of fast-type $\mathrm{M}$. gastrocnemius cytes after $\sim 30$ days of dox-treatment, while yet-unaffected MUT cells still showed the regular, WT-like sarcomere pattern (Fig. 3g). In affected myocytes, we observed the following alterations: (1) Sarcomere disintegration, typically beginning at the I-bands, including dissociation of sarcomeric Z-discs and Abands, which became misaligned relative to one another. The organization into I-, A-, and M-bands disappeared. (2) Loss of 
Fig. 3 Ultrastructural changes in dox-treated MUT vs. WT gastrocnemius myocytes. a Anti-TTN antibody epitope locations and TTN variants. b Confocal images of immunolabeled longitudinal sections of WT and MUT muscles after 30 days of dox-treatment, using antibodies to titin Z/I-band (2080; red) and $\alpha$-actinin (ACTN2, green); nuclear staining (Hoechst, blue) shown on merge. c Percentage of cells without cross-striations in WT and MUT gastrocnemius muscles after 21 days ( $<10 \%$ body weight loss, BWL) or $>28$ days of dox-treatment $(>10 \% \mathrm{BWL}$ ), counted on images stained against Ttn-Z/I-2080 and ACTN2; $n=3$ animals/group. d-f Confocal images of immunolabeled longitudinal sections of WT and MUT muscles after 30 days of dox-treatment, using antibodies to various TTN species (red) and ACTN2 (green) or myosin heavy chain (MyHC, green); nuclear staining by Hoechst (blue) is also shown on merged images. d) TTN Novex3 and MyHC co-staining. e) TTN Cronos and ACTN2 co-staining. f) TTN kinase domain near M-band (M-TtnK) and ACTN2 co-staining. In $\mathbf{b}$ and $\mathbf{d}-\mathbf{f}$, secondary antibodies are Cy3- or fluorescein-conjugated anti-rabbit or anti-mouse lgG. Bars, $25 \mu \mathrm{m}$; insets show detail at $3 \mathrm{x}$ magnification. $\mathbf{g}$ Electron micrographs of WT (left panel) and MUT muscles after 30 days of dox-treatment. Z, Z-disc; I, I-band; A, A-band; M, M-band. Right panel shows average circumference of sagittal Z-disc planes, measured on electron micrographs of doxycycline-treated WT and MUT mouse ( $>10 \%$ BWL) muscles ( $n=2$ animals/group and 250 Z-discs/group). $\mathbf{h}$ Immunoelectron micrographs using antibodies to ACTN2. Inset shows detail at $\sim 2.5 \mathrm{x}$ magnification. i-m Immunoelectron micrographs using antibodies to four different TTN epitopes. $\mathbf{i}$ Antibody epitopes in WT. $\mathbf{k}$ Localization of TTN Z/Iband junction (TTN Z/I) by 2080 and T12 antibodies in MUT. I Localization of TTN I/A-band junction by MIR antibody (I/A-MIR). $\mathbf{m}$ Localization of TTN M-band region by TtnK antibody (M-Ttnk). In $\mathbf{g}$ - $\mathbf{m}$, secondary antibodies are nanogold-conjugated anti-mouse or anti-rabbit lgGs; bars, $1 \mu \mathrm{m}$. Data in $\mathbf{c}$ and $\mathbf{g}$ are mean $\pm \mathrm{SD} ;{ }^{\star \star \star \star} p<0.0001 ;{ }^{\star \star} p<0.01$, in unpaired, two-tailed Student's $t$-test (c) or two-tailed Mann-Whitney test (g). Immunofluorescence micrographs shown are representative of 2-5 images/condition, electron micrographs of $>25$ images/condition. Source data are provided as a Source Data file.

dox-treatment (Supplementary Fig. 6a), although at a much lower degree.

Immunogold-EM demonstrated that Z-disc-like rods/aggregates in MUT cells were ACTN2-positive (Fig. 3h). Antibodies to various TTN regions (Fig. 3i) revealed that epitopes at the Z/Ijunction (2080, T12) adopted a diffusely spread, sparse pattern in MUT cells, suggesting that this TTN region had become uncoupled from the remnant sarcomere-like structures and was rapidly lost (Fig. 3k). A similar pattern of sarcomere disintegration was frequently seen with antibodies to the TTN I/A-junction (I/A-MIR) and M-band (M-TtnK) (Fig. 3l, m). Interestingly, we occasionally observed isolated A-bands in MUT gastrocnemius, which were stained at their edges by I/A-MIR and at their center by $\mathrm{M}-\mathrm{TtnK}$ (Fig. 31, m). Such A-bands could appear completely detached from the Z-rods/aggregates, whereas sometimes, the Zaggregates emerged in the middle of an A-band. These findings confirm that TTN-inactivation causes gradual loss of sarcomere integrity and promotes abnormal protein-protein interactions, including Z-disc-protein aggregation and streaming.

TTN-based sarcomere disassembly impedes myofiber mechanics. Next, we sought to test whether loss of TTN affects myofiber mechanical properties. We first measured the lateral stiffness by transverse nanoindentation using atomic force microscopy (Fig. 4a). Single myofibers were dissected from doxtreated WT and MUT (TTN-loss, $>80 \%$ ) muscles, skinned, and attached to glass-bottom dishes coated with Cell-Tak glue in a relaxing buffer (including $\mathrm{Mg}^{2+}$-ATP but excluding calcium). Force-indentation curves recorded on myofibers of pectoralis muscle showed significant softening in MUT. The Young's modulus (lateral stiffness) decreased from $7.89 \pm 4.65 \mathrm{kPa}$ in WT to $3.92 \pm 3.06 \mathrm{kPa}$ in MUT, whereas the indentation depth at 3 $\mathrm{nN}$ indentation force increased from $268.5 \pm 160.2 \mathrm{~nm}$ in WT to $461.4 \pm 267.5 \mathrm{~nm}$ in MUT (mean \pm SD; $n=119$ ) (Fig. 4b). Tensile stiffness measured during stepwise stretching of skinned vastus lateralis fibers in relaxing buffer decreased by $75 \%$ or more in MUT vs. WT (Fig. 4c). Specific tension of $\mathrm{Ca}^{2+}$-activated myofibers was reduced on average by $\sim 70 \%$ (Fig. $4 \mathrm{~d}$ ). Thus, the TTNloss increases myofiber indentability, lowers transversal stiffness, and reduces both passive and active tension.

TTN depletion causes loss of myosin but not actin and Cronos. In $\mathrm{M}$. pectoralis from mice treated with doxycycline for at least 4 weeks, full-length TTN protein N2A was reduced in MUT vs. WT by $54 \pm 15 \%$ (difference between mean \pm SEM) relative to actin protein (Fig. 5a). No consistent changes in actin appeared in MUT and also the NEB/actin ratio was not significantly altered (Fig. 5a). The MyHC/actin ratio was reduced by $45 \pm 7 \%$, whereas the TTN/MyHC ratio was unaltered, in MUT vs. WT (Fig. 5a). Similar results were obtained for other fast-type skeletal muscles. Western blotting of M. gastrocnemius using antibodies to MyHC$\mathrm{IIb}$, the predominant isoform in fast mouse muscles ${ }^{29}$, demonstrated downregulation in MUT by $34 \pm 12 \%$ (Fig. 5b). At the transcriptional level, myosin-heavy-chain-4 (Myh4) coding for MyHC-IIb and alpha-1 skeletal-muscle actin (Acta1) were not significantly altered in MUT vs. WT, although there was a trend toward reduction in MUT (Fig. 5c). On immunoblots of gastrocnemius or pectoralis muscles, the N2A and Novex-3 isoforms were significantly downregulated in MUT, by $62 \pm 23 \%$ and $82 \%$ (Hodges-Lehmann estimator), respectively (Fig. 5d). Cronos remained unchanged on average, although the expression level varied greatly in MUT.

Similar analyses performed in muscles exposed to doxycycline for 21 days revealed still-preserved N2A/actin and N2A/GAPDH protein ratios in MUT (Supplementary Fig. 6b, c), although Nterminal titin exons were effectively suppressed (Supplementary Fig. 6d); however, Novex-3 protein was already missing (Supplementary Fig. 6c). Cronos TTN was preserved at both protein and transcript levels. Interestingly, the MyHC/actin protein ratio was already reduced in MUT, as was Myh4 mRNA, whereas actin transcripts (Acta1) were unaltered (Supplementary Fig. 6b, d). In summary, loss of Z-disc-anchored TTN caused reduction of myosin but not thin-filament proteins.

Cronos stabilizes A-bands but does not preserve sarcomeres. The presence of disconnected A-bands (Fig. 31, m) and preservation of Cronos expression (Figs. 1e and 5d, Supplementary Fig. 6) in MUT myocytes suggested that remnant A-bands may be stabilized by Cronos. To test this hypothesis, we studied fatigueresistant skeletal muscles, because we found that in these muscles - specifically, soleus and diaphragm-Cronos protein is not expressed at detectable levels, contrasting the abundant Cronos transcript and protein expression in fast skeletal muscles and the heart (Fig. 6a, b). Immunoblot analysis of TTN in dox-treated WT and MUT diaphragm demonstrated reduction of N2A and Novex-3 in MUT and confirmed the lack of Cronos expression (Fig. 6c, d). Similar to fast-type muscles, the NEB/actin ratio remained unaltered, whereas the $\mathrm{MyHC} /$ actin ratio was significantly lower in MUT $(1.50 \pm 0.10$; mean \pm SD) than in WT $(1.90 \pm 0.09)$ (Fig. $6 \mathrm{~d})$, although the relative reduction was less 
a

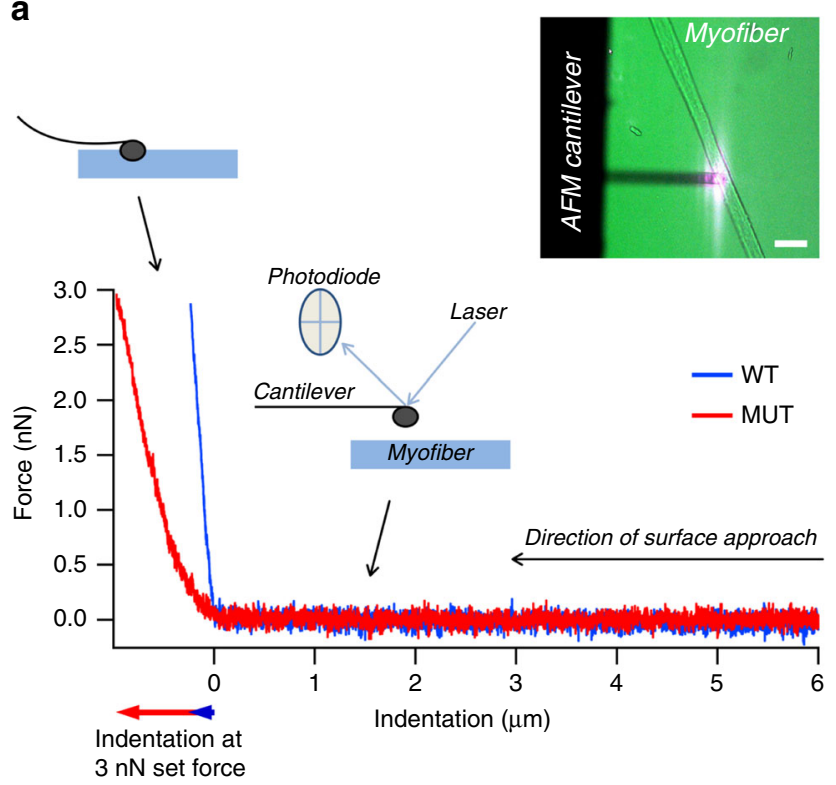

C

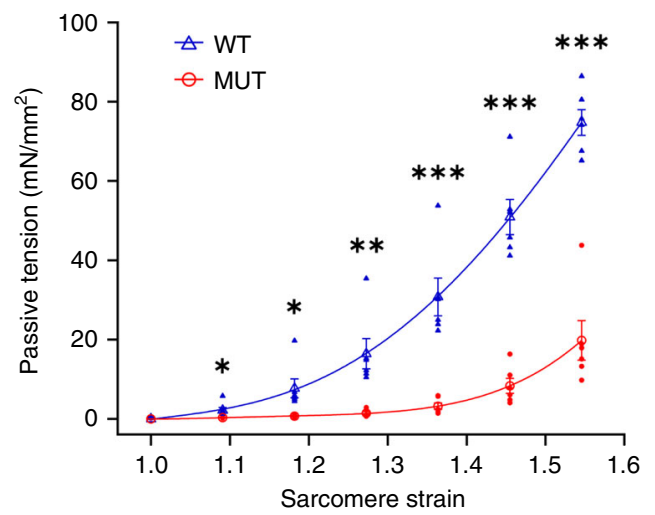

b
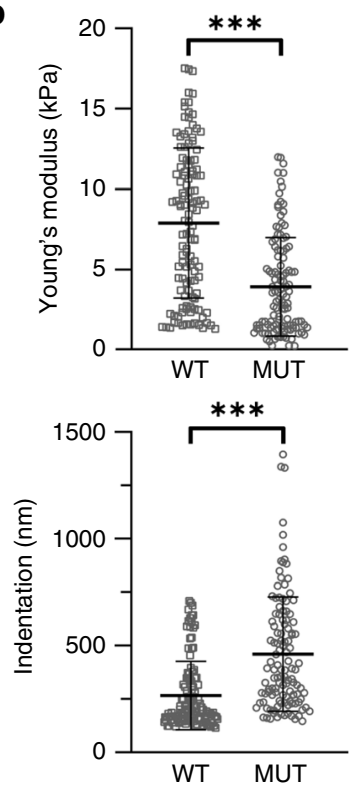

d

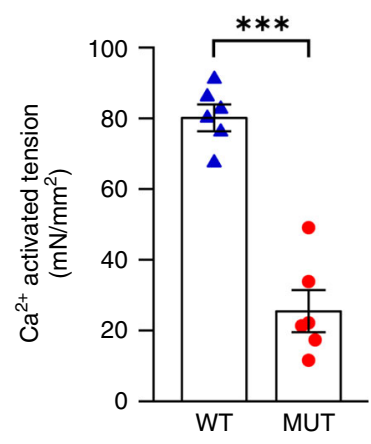

Fig. 4 Myofiber stiffness and tension from WT and MUT mice after $\mathbf{3 0}$ days of dox-treatment. a Representative force-indentation curves for myofibers from WT (blue) and MUT (red) mouse pectoralis muscles after dox-treatment. Schematics indicate the principle of measurement by atomic force microscopy (AFM), whereby the cantilever approaches the cell surface until it begins to indent the cell at indentation 0 . Indentation was quantified at a pre-set force value of $3 \mathrm{nN}$. The Young's modulus was calculated by assuming a linear slope of the force-indentation curve at forces above $\sim 0.5 \mathrm{nN}$. Inset, image of a single myofiber glued to the cover glass with Cell-Tak and indented by the AFM cantilever. Bar, $100 \mu \mathrm{m}$. b Young's modulus and indentation depth (at $3 \mathrm{nN}$ indentation force) for WT and MUT myofibers treated with doxycycline. $n=119$ recordings/condition; $n=6 \mathrm{~m}$. pectoralis fibers were prepared from $n=2$ different mice per group. c Passive force of isolated, skinned, vastus lateralis WT and MUT myofibers at different stretch states, normalized to cross-sectional area ( $n=6$ fibers from $n=3$ different mice/group). Strain was estimated from sarcomere-length (SL) measurements by laser diffraction (strain 1.0, slack length of $\sim 2.2 \mu \mathrm{m} \mathrm{SL}$; strain $1.55, \sim 3.4 \mu \mathrm{m} \mathrm{SL}$ ). Fits are polynomial regressions. d) Specific developed tension of vastus lateralis myofibers in activation buffer with $10^{-4.5} \mathrm{~mol} / \mathrm{L}$ free calcium ( $n=6$ fibers from $n=3$ different mice/group). Skinned myofibers were stretched (strain, 1.2; 2.6 $\mu \mathrm{m} \mathrm{SL}$ ) in relaxing solution, held for $2 \mathrm{~min}$, and then maximally activated by calcium. Average data are mean \pm SD in $\mathbf{b}$ and mean $\pm \mathrm{SEM}$ in c and $\mathbf{d} ;{ }^{* \star *} p<0.001,{ }^{\star \star} p<0.01$, in unpaired, two-tailed, Student's t-test. Source data are provided as a Source Data file.

than in fast-type gastrocnemius muscle (cf., Fig. 5a). Importantly, electron micrographs demonstrated the simultaneous disassembly of all parts of the sarcomere, including the A-band, in MUT diaphragm myocytes (Fig. 6e). While remnant A-bands were never observed, Z-aggregates and Z-disc streaming were frequent. We conclude that Cronos is responsible for transient survival of some A-bands during loss of Z-disc-anchored TTN in fast-type muscles. The lack of Cronos in fatigue-resistant muscles may underlie the general decline of sarcomeric structure in MUT diaphragm. However, even in fast muscles, Cronos at best can delay sarcomere deterioration.

TTN ablation is accompanied by increased TTN ubiquitination. In situ hybridization and $\mathrm{qPCR}$ measurements demonstrated that very little full-length TTN mRNA remained in MUT muscles after 21-30 days of dox-treatment. Hence, TTN proteins will not be replaced and remaining TTN will eventually be targeted for degradation by the ubiquitination pathway ${ }^{30}$. Analysis of TTN-ubiquitination in fast-type muscle using antibodies to all ubiquitin species (pan-Ub) revealed increased signals for remaining $\mathrm{N} 2 \mathrm{~A}(\sim 3.6 \mathrm{MDa})$ and Novex-3 ( 0.7 MDa) in doxtreated MUT (Fig. 7a). Interestingly, increased ubiquitination in MUT was much more pronounced for the smaller Cronos/T2 TTN species. The proteolytic T2-fragment of TTN ( 2.4 MDa) co-migrates with Cronos $(\sim 2.3 \mathrm{MDa})$ on titin gels ${ }^{31}$. No panubiquitination of any TTN species was seen in dox-treated WT. Proteins smaller than TTN were more highly ubiquitinated in MUT than in WT (Fig. 7a). In summary, TTN-inactivation causes increased ubiquitination of remaining TTN protein and other myocyte proteins. 
a

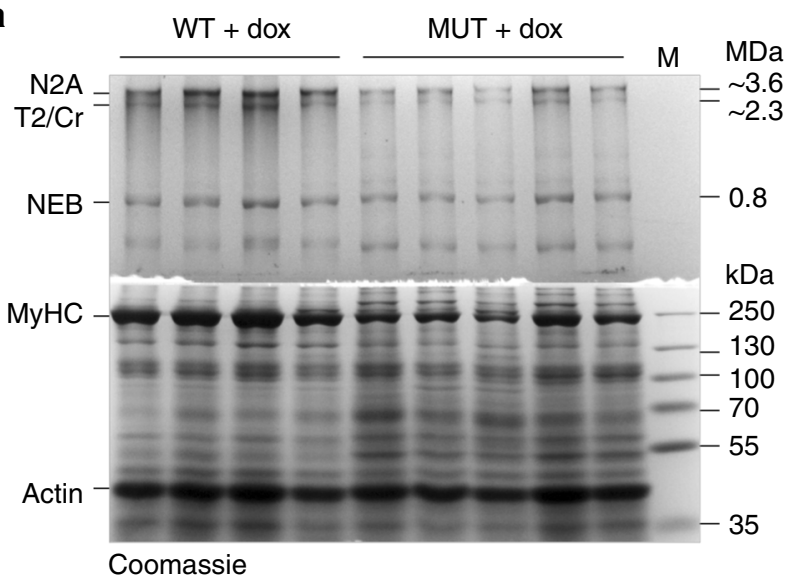

b

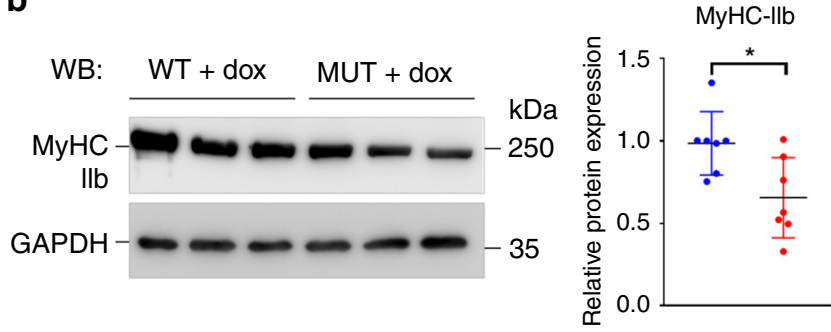

TTN N2A/Actin
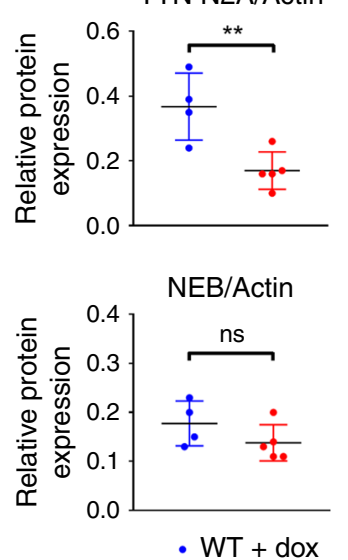

MyHC/Actin

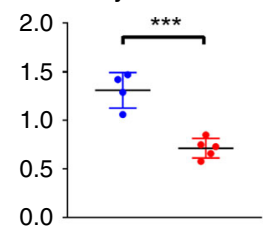

TTN N2A/MyHC

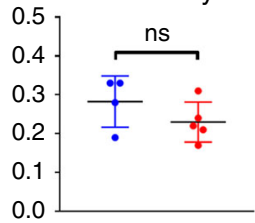

- MUT + dox

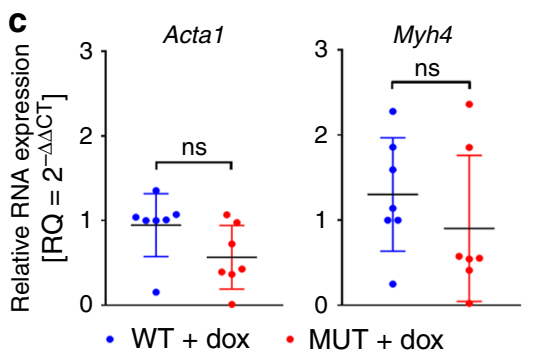

d
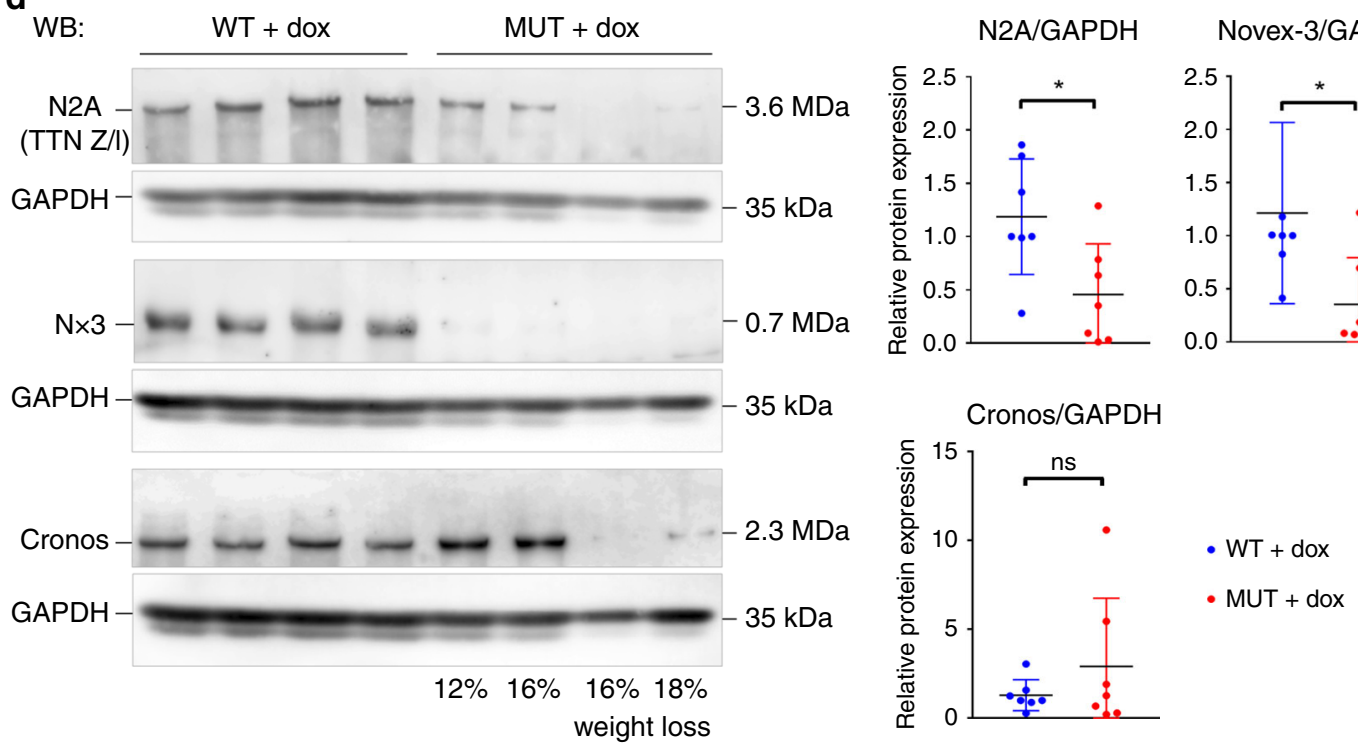

Fig. 5 Loss of Z-disc anchored TTN reduces myosin but not actin or Cronos. a Coomassie-stained titin gel (2.5\% polyacrylamide) stacked onto a 10\% running gel and loaded with protein lysates from dox-treated WT and MUT Ttn ${ }^{\text {tm1c; }}$;CTA1-rtTA;tetO-cre pectoralis muscle. Right panels show results of densitometric quantification, comparing TTN (N2A), MyHC, or NEB expression to actin levels, as well as TTN (N2A) to MyHC levels, from WT ( $n=4)$ and MUT $(n=5)$ mice. NEB, nebulin; MyHC, myosin heavy chain; T2, titin proteolytic fragment; $\mathrm{Cr}$, Cronos isoform; $M$, marker. ${ }^{\star \star} p=0.0081 ;{ }^{* \star \star} p=0.0004$; ns, non-significant. b Western blots detecting MyHC-Ilb protein in MUT vs. WT gastrocnemius muscles. Right panel shows mean MyHC-Ilb:GAPDH ratios in WT (blue) and MUT (red); $n=7$ mice/group; ${ }^{\star} p=0.0158$. c Relative mRNA expression of alpha 1 skeletal muscle actin (Acta1) and Myh4 (codes for MyHC-Ilb) in gastrocnemius muscles measured by qPCR; $n=7$ mice/group; ns, non-significant. N2A titin was probed by antibodies to TTN Z/I (2080). d Representative western blots showing expression of TTN isoforms in WT and MUT Ttn ${ }^{\text {tm1c; } A C T A 1-r t T A ; t e t O-c r e ~ p e c t o r a l i s ~ m u s c l e . ~ G A P D H ~ s e r v e d ~ a s ~}$ loading control. Nx3, Novex-3 isoform. Right panels show results of densitometric quantification of western blots performed on WT and MUT gastrocnemius muscles, indexed to WT levels $\left(n=7\right.$ mice/group); ${ }^{\star} p_{\mathrm{N} 2 \mathrm{~A}}=0.0201 ;{ }^{\star} p_{\text {Novex }-3}=0.0239$. All graphs show mean \pm SD. All $p$-values were calculated using two-tailed unpaired Mann-Whitney U-test or two-tailed unpaired Student's t-test. All data were obtained from $\sim 30$-day dox-treated muscles. Source data are provided as a Source Data file. 
a

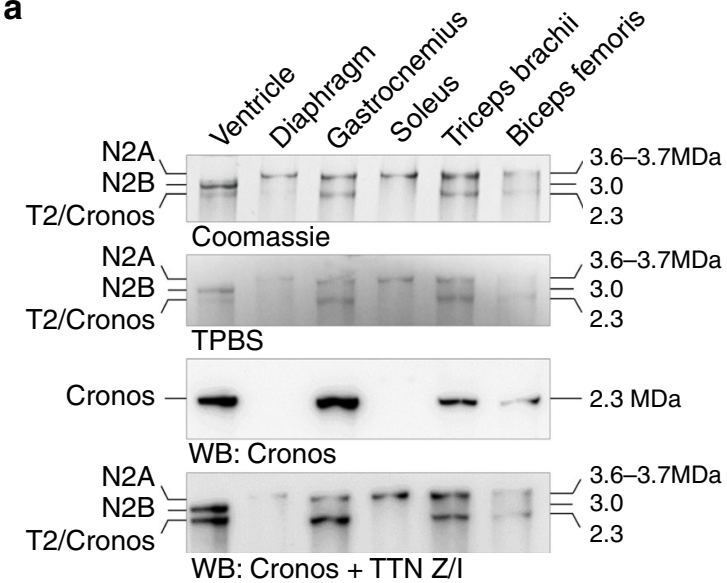

c

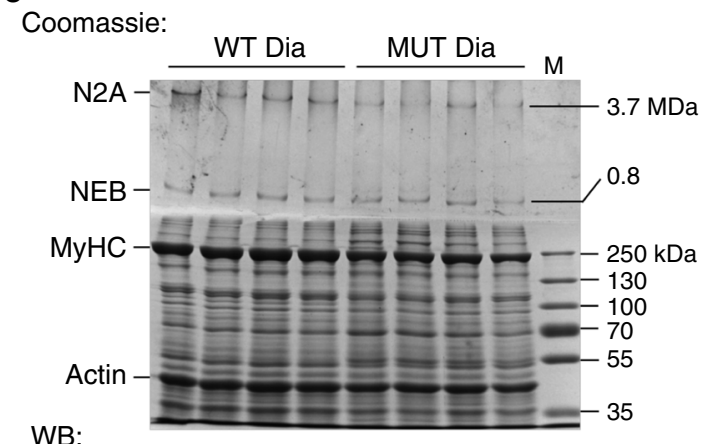

WB:
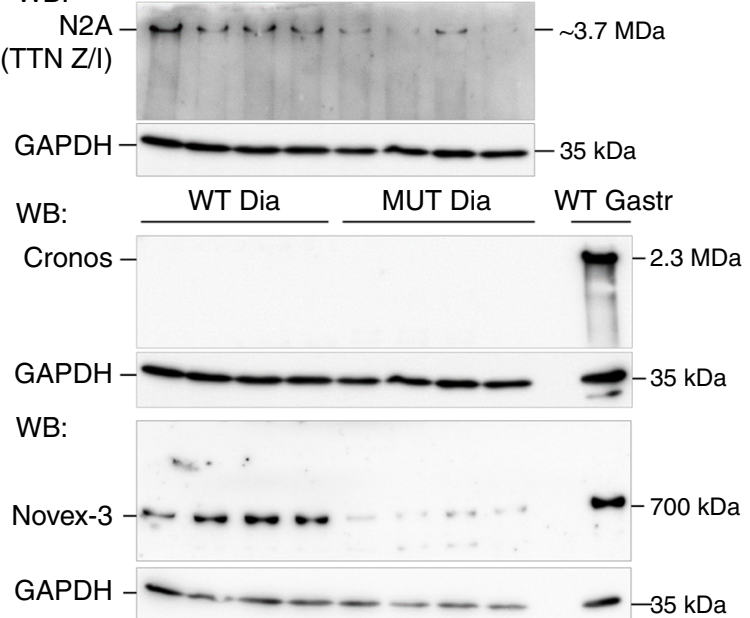

b

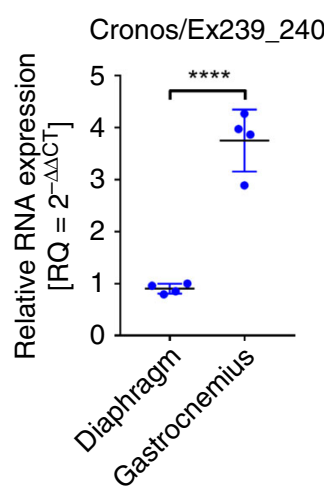

d

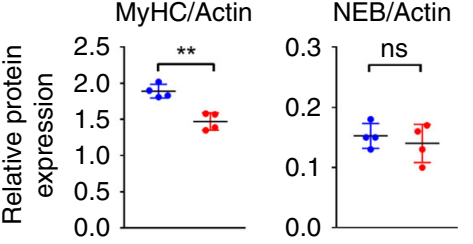

TTN N2A/GAPDH Novex-3/GAPDH

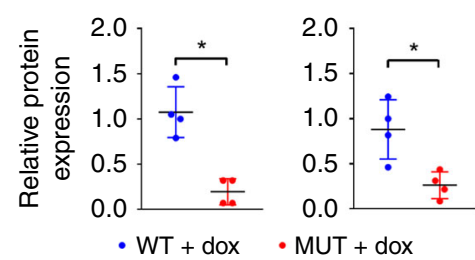

e
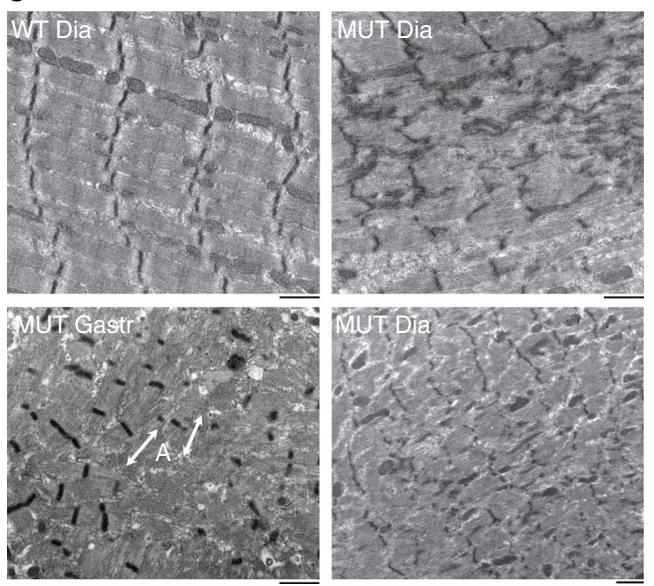

Fig. 6 Differences in Cronos expression and A-band stability between muscle types. a Expression of full-length TTN and Cronos in different muscle types of wild-type mice. Top panel, Coomassie-stained, 2\% SDS-polyacrylamide gel; lower two panels, western blots (WB) using antibodies to Cronos or both Cronos and full-length TTN (antibody 2080 against TTN Z/I). TPBS, total protein stain of blotted TTN proteins. Gels and blots shown are representative of 2-3 gels/WBs performed per condition. b Relative Cronos mRNA expression in wild-type mouse muscles measured by qPCR; $n=4$ mice/group. ${ }^{\star \star \star \star} p<$ 0.0001 , by two-tailed unpaired Student's $t$-test. c Coomassie-stained titin gel ( $2.5 \%$ polyacrylamide) stacked onto a $10 \%$ running gel and loaded with protein lysates from WT $(n=4)$ and MUT $(n=4)$ Ttn ${ }^{\text {tmlc; }}$ ACTA1-rtTA;tetO-cre diaphragm muscle after dox-treatment (top panel). M, marker. Lower panels show representative western blots detecting expression of TTN isoforms in dox-treated WT and MUT diaphragm muscles. Full-length titin (N2A) was probed by antibodies to TTN Z/I (2080). GAPDH served as loading control. d Results of densitometric quantification ( $n=4$ mice/group); MyHC/ Actin and NEB/Actin ratios were measured on Coomassie-stained titin gel, N2A, and Novex-3 expression on western blots, relative to GAPDH. Data are mean $\pm S D ;{ }^{\star \star} p=0.0016,{ }^{\star} p_{\mathrm{N} 2 \mathrm{~A}}=0.0286,{ }^{\star} p_{\mathrm{Novex} 3}=0.014, \mathrm{~ns}$, non-significant; calculated using a two-tailed unpaired Student's $t$-test. e Representative electron micrographs (out of $>20$ images recorded per condition) of WT (upper left panel) and MUT (right panels) mouse diaphragm muscles after doxtreatment. For comparison, the lower left panel shows a dox-treated MUT gastrocnemius myocyte. Arrows labeled A point to remnant, isolated A-bands. Bars, $1 \mu \mathrm{m}$. All data were obtained after $\sim 30$ days of dox-treatment. Source data are provided as a Source Data file. 
a

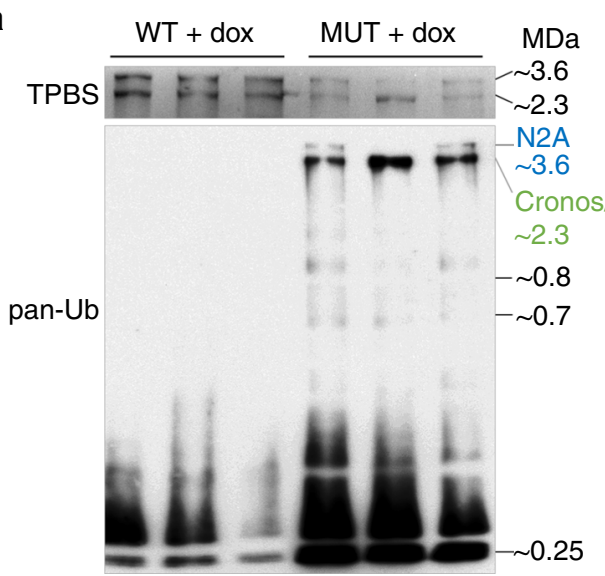

b

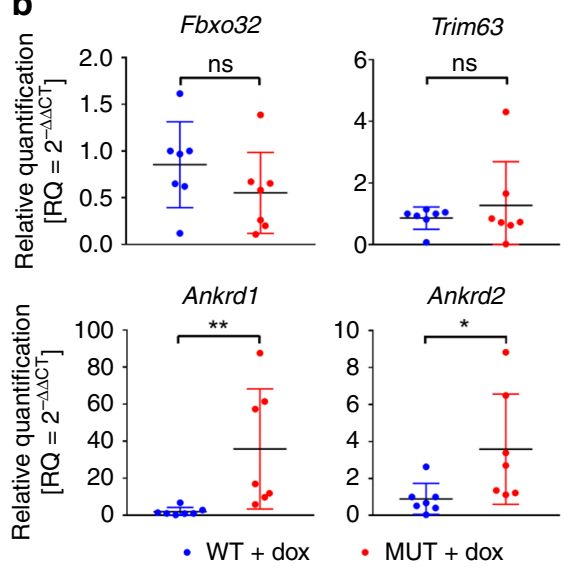

C

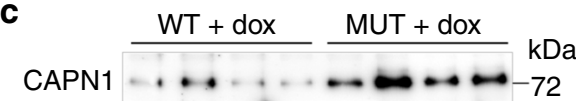

$\mathrm{GAPDH} \sim-\infty-\infty--35$

CAPN3
CAPN1/2 $-\vdots:=\Xi \Xi \Xi-75$
-72

GAPDH $-\infty-\infty-\infty-35$

HSP90 $--\leq-\infty----95$

GAPDH - - - - - - - -

SMYD1 ..... -72

GAPDH $-\cdots=-\cdots-35$

SQSTM1 $\rightarrow==\cdots-\cdots-_{-55}$

GAPDH $\rightarrow--\infty-\infty--35$

LAMP2 $=-m=\mathbf{0} \mathbf{- 0}-100$

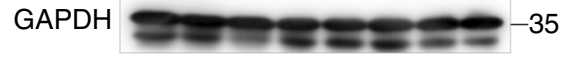

d

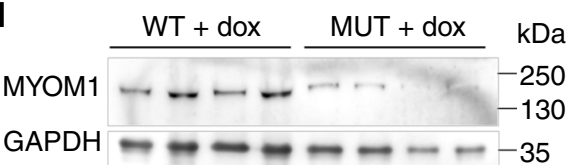

ACTN2 - - - - - -95

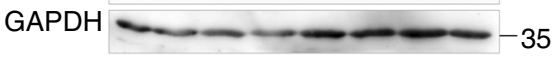

T-CAP $\cdots \cdots \cdots$

$\mathrm{GAPDH}-\longrightarrow-17$

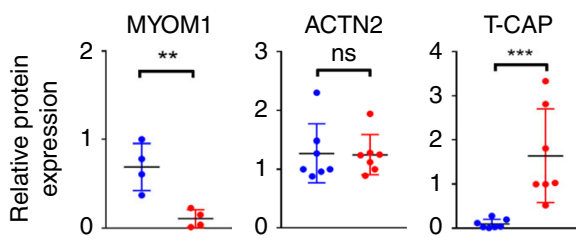

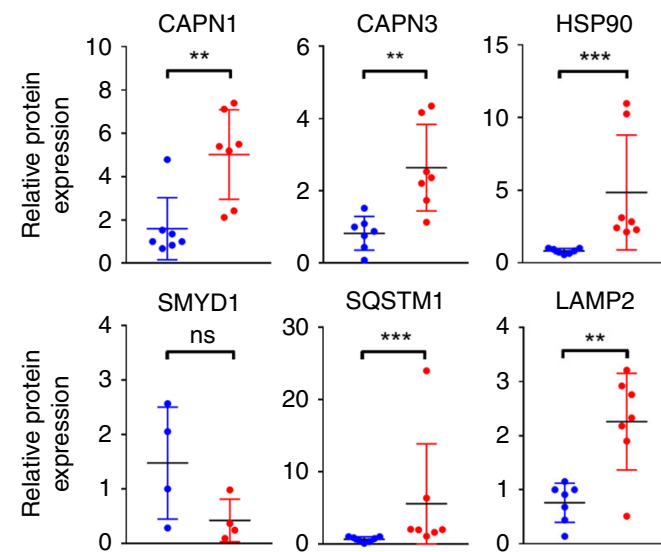

- $\mathrm{WT}+$ dox $\quad$ MUT + dox

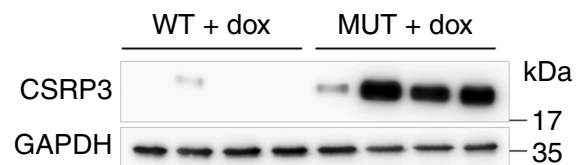

ANKRD1 $---= \pm=$

TPBS

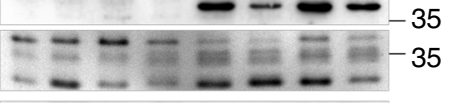

ANKRD2

GAPDH $=-\cdots=-35$

CSRP3 ANKRD1 ANKRD2

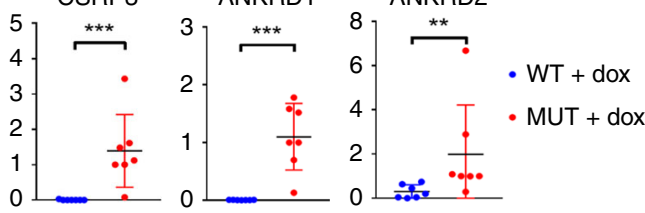

Loss of TTN activates the PQC machinery. Sarcomere-bound E3-ubiquitin ligases have been implicated in muscle atrophy, particularly Z-disc-associated Fbxo32 (MAFbx/atrogin-1) and Mband-associated (TTN-binding) Trim63 (MuRF1) ${ }^{32}$. However, none of these molecules was significantly altered at transcript level in dox-treated MUT vs. WT M. gastrocnemius (Fig. 7b). In contrast, two calcium-dependent cysteine proteases, ubiquitously expressed calpain-1 (CAPN1) and skeletal muscle-specific calpain-

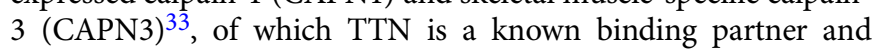
substrate, were significantly increased at protein level in MUT vs. WT (Fig. 7c). As expected, anti-CAPN3 antibodies recognized the $94-\mathrm{kDa}$ CAPN3 protein and an additional $\sim 80 \mathrm{kDa}$ band 
Fig. 7 Effects of TTN-loss on PQC markers and TTN-binding mechanosensitive proteins. a Western blot (2.5\% polyacrylamide gel) of gastrocnemius muscle from dox-treated wild-type (WT) and mutant (MUT) mice, using antibodies to pan-ubiquitin (pan-Ub). TPBS, total protein stain of blotted TTN proteins. The blot shown is representative of 2 similar blots performed. $\mathbf{b}$ Expression of E3 ubiquitin ligases Fbxo32 and Trim63 in dox-treated WT and MUT muscles at transcript level by real-time quantitative RT-PCR. In addition, transcript levels for TTN-binding proteins Ankrd1 and Ankrd2 are shown. Data are mean \pm SD ( $n=7$ mice/group). c Expression of PQC markers calpain (CAPN1; CAPN3), heat shock protein 90 (HSP90) and co-chaperone Smyd1, sequestosome-1 (SQSTM1), and lysosome-associated membrane protein 2 (LAMP2) by western blot. Right panels show results of densitometric quantification; $n=7$ mice/group, except for SMYD1 $(n=4)$. d Expression of TTN-binding proteins myomesin-1 (MYOM1), $\alpha$-actinin (ACTN2), telethonin (T-CAP), muscle LIM protein (CSRP3), Ankrd1 and Ankrd2, by western blot. Lower panels show results of densitometric quantification; $n=7$ mice/group, except for MYOM1 ( $n=4$ ). For quantification of signals on western blots, GAPDH served as a reference. ${ }^{\star \star \star} p<0.001,{ }^{\star \star} p<0.01$, ns, non-significant; two-tailed Mann-Whitney test (Trim63, Ankrd1, CAPN1, HSP90, SQSTM1, LAMP2, ACTN2, T-CAP, CSRP3, ANKRD2) or unpaired, two-tailed Student's t-test (Fbxo32, Ankrd2, CAPN3, SMYD1, MYOM1, ANKRD1). All data were obtained after $\sim 30$ days of dox-treatment. Source data are provided as a Source Data file.

corresponding to CAPN1 and/or CAPN2; 34 all these calpain species were upregulated in MUT tissue. Chaperones important for the assembly/maintenance of myosin and TTN were also altered: heat shock protein (HSP) 90 was significantly increased in MUT (Fig. 7c), as was the small HSP alpha- $\beta$-crystallin (CRYAB; Supplementary Fig. 7), whereas the HSP90 co-chaperone Smyd1 (also important for myosin assembly) ${ }^{35}$ showed a strong trend toward reduction in MUT (Fig. 7c). The chaperones HSP70 and HSC70 remained unchanged (Supplementary Fig. 7). Furthermore, the autophagy adapter protein sequestosome-1 (SQSTM1/ p62), which also associates with M-band $\mathrm{TTN}^{36}$, was significantly increased at protein level in MUT vs. WT muscles (Fig. 7c). Likewise, we found significant upregulation of lysosomeassociated membrane-protein-2 (LAMP2), which is involved in lysosomal stability and autophagy. In summary, TTN-loss activated crucial factors important for muscle protein degradation and turnover, including relevant chaperones, proteasomal and autophagy-lysosomal pathways.

TTN-binding mechanosensitive proteins increase with TTN loss. The high intensity of ACTN2 signals despite the disruption of regular ACTN2 striations in TTN-depleted muscles (Fig. 3) suggested mislocalization/aggregation rather than loss of the Z-disc protein. Indeed, ACTN2 protein expression was not significantly altered in dox-treated MUT vs. WT (Fig. 7d). In contrast, the Mband protein myomesin-1 (MYOM1) was highly reduced in MUT. The N-terminus of TTN complexes not only with ACTN2, but also with telethonin (T-CAP) and cysteine-and-glycine-rich protein-3 (CSRP3; also known as muscle LIM protein, MLP), which has been proposed to act as a mechanosensor ${ }^{37}$. We found that loss of $\mathrm{Z}$ disc-anchored TTN markedly increased T-CAP and CSRP3 protein levels (Fig. 7d). Other titin-binding proteins, four-and-a-half-LIM domain (FHL) 1 and $2^{38,39}$, showed either no significant change (FHL2) or the opposite regulation of two isoforms (FHL1) (Supplementary Fig. 7). Additional molecules associating with I-band TTN include the muscle ankyrin-repeat proteins (MARPs) ${ }^{40,41}$. The expression of ankyrin-repeat-domain-1 (Ankrd1/CARP) and ankyrin-repeat-domain-2 (Ankrd2) was highly increased in doxtreated MUT vs. WT muscles, at both transcript (Fig. 7b) and protein levels (Fig. 7d).

TTN loss occurs in critical illness myopathy patient muscles. The progressive loss of thick- but not thin-filament proteins after TTN-inactivation is reminiscent of a special form of human muscle-wasting disease known as CIM, acute quadriplegic myopathy, or myosinopathy ${ }^{26}$. Typically, affected individuals are critically ill ICU patients on long-term mechanical ventilation support, who present with generalized muscle weakness or paralysis due to rapid atrophy of peripheral muscles. Unfortunately, the causes for myosinopathy are poorly understood obfuscating treatment. We confirmed the progressive loss of myosin protein in biopsies of tibialis anterior (TA) muscles from CIM patients (Fig. 8a) and classified the biopsies into groups with high MyHC:actin (My:Ac) (mean $\pm \mathrm{SD}, 2.10 \pm 0.44 ; n=5$ individuals), intermediate (1.39 \pm $0.25 ; n=5)$, and low expression ratios $(0.70 \pm 0.34 ; n=4)$ (Fig. $8 \mathrm{a})$. The actin-expression levels remained constant even in severely affected CIM muscles (Fig. 8a, Supplementary Fig. 8).

Interestingly, we found strongly reduced TTN:actin ratios in muscles with intermediate- or low-MyHC content, compared to those with high-MyHC content (mean \pm SD, $0.71 \pm 0.23$ (high My: Ac) vs. $0.45 \pm 0.15$ (intermediate $\mathrm{My:Ac}$ ) vs. $0.39 \pm 0.21$ (low My: Ac)) (Fig. 8a, Supplementary Fig. 8). The difference in relative TTN expression was not significant between the intermediate- and lowMy:Ac groups, suggesting that most of the TTN-loss occurred at earlier stages of muscle atrophy. As a consequence, the average TTN:MyHC ratio was similar in the high-MyHC (mean $\pm \mathrm{SD}, 0.35$ $\pm 0.14)$ and intermediate-MyHC groups $(0.32 \pm 0.06)$, but significantly increased in the low-MyHC group $(0.58 \pm 0.23)$ (Fig. 8a). We also found elevated levels of (pan-)ubiquitinated TTN in CIM muscles with low My:Ac ratios, compared to those with high ratios (Fig. 8b), suggesting increased degradation.

Both the essential and the regulatory myosin light chains were consistently reduced or missing in low-My:Ac CIM muscles (Supplementary Fig. 8a). Another A-band protein, myosinbinding protein-C (MyBPC), was progressively lowered: the MyBPC:actin ratio changed from $0.29 \pm 0.07$ (high My:Ac; mean $\pm \mathrm{SD}$ ) to $0.19 \pm 0.07$ (intermediate My:Ac) and $0.09 \pm 0.05$ (low My: Ac) (Fig. 8a). Furthermore, MYOM1 was nearly absent in CIM muscles with low My:Ac ratios (Fig. 8c). In contrast, nebulin showed little changes with decreasing My:Ac ratios (Fig. 8a). Expression of ACTN2 was identical in muscles with high and intermediate $\mathrm{My}: \mathrm{Ac}$ ratios, but modestly increased in those with low My:Ac ratio (Fig. 8a). These alterations in sarcomere proteins in CIM patient muscles were accompanied by increased expression levels of other titin-binding molecules in the low-My:Ac vs. high My:Ac groups, which included the chaperone HSP90 (but not HSP70), ANKRD1, and FHL2 (Fig. 8d). Importantly, the changes observed in CIM muscles are (at least qualitatively) highly similar to those in mouse muscles after TTN-inactivation.

Electron microscopy confirmed degradation of thick filaments and preservation of Z-discs and thin filaments in TA muscles from CIM patients with low My:Ac ratios (Fig. 8e). Various changes in the structural organization of myocytes from low-My:Ac-CIM muscles were akin to those observed in TTN-depleted fast-type MUT mouse muscles, including Z-disc deformations and loss of myofibril orientation (cf. Fig. 3g). Taken together, our findings suggest that TTN-loss is a prominent feature of adult muscle remodeling in CIM. In concert with TTN-associated proteins, TTN may act as a key mechanosensor, whose (partial) absence strongly contributes to the rapid muscle atrophy in CIM patients.

\section{Discussion}

Not many details are known about the life cycle of adult muscle sarcomeres, including their maintenance and turnover of proteins. This issue is relevant, because differentiated myocytes are 

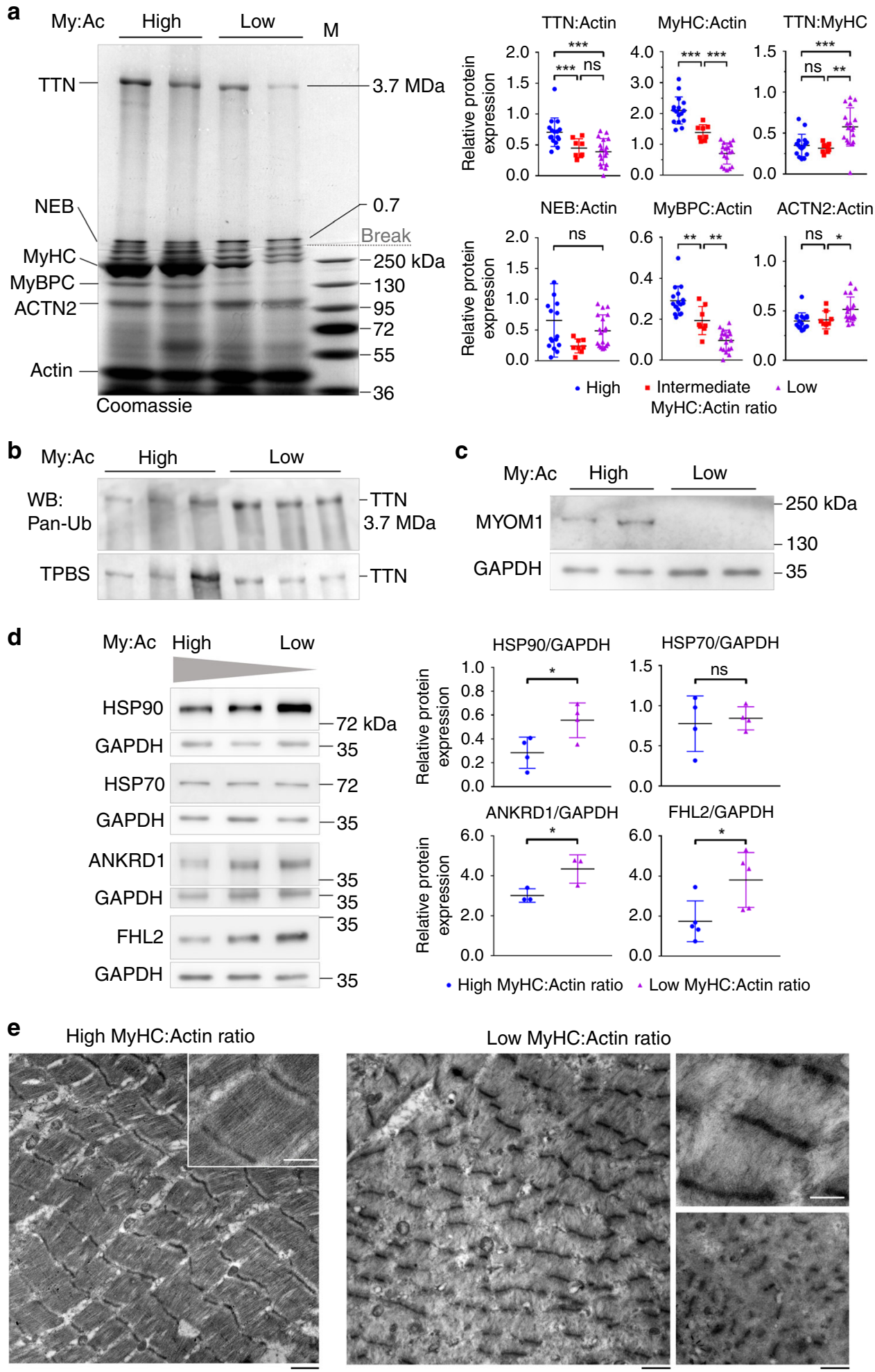

unable to renew and repair themselves by cell division and must continuously replace their building parts, including the sarcomeric proteins, whose lifetime is limited. Since TTN is considered the backbone of the sarcomere, we sought to determine how this protein is involved in the maintenance of sarcomeric integrity by removing the Z-disc-anchored isoforms in adult murine skeletal muscle. We observed a dramatic loss of muscle function beginning $\sim 3$ weeks after the supply of new TTN stopped, concomitant with the gradual disintegration of the sarcomeres, which is consistent with a TTN half-life of $2-3$ weeks ${ }^{42}$. TTN-loss caused reduction of thick- but not thin-filament proteins, myocyte mechanical impairment, activation of PQC systems, and increased expression of TTN-binding mechanosensory proteins. These findings clearly indicate that Z-disc-anchored TTN is crucial for sarcomere stability and function in adult muscle. Remarkably, we find that progressive loss of full-length TTN 
Fig. 8 Protein expression and ultrastructure of CIM patient muscles. a Left panel shows a representative Coomassie-stained titin gel (1.8\% polyacrylamide) stacked onto a $6 \%$ running gel and loaded with biopsy samples of tibialis anterior muscles from critical illness myopathy (CIM) patients expressing high or low myosin heavy chain $(\mathrm{MyHC})$ over actin ( $\mathrm{My}: \mathrm{Ac}$ ) ratios. Right panels show results of densitometric analysis of $\mathrm{Coomassie-stained}$ stacking gels. Quantification of relative sarcomeric protein expression was done separately for CIM muscles with high $(n=5$ patients), intermediate ( $n=5$ patients), and low ( $n=4$ patients) My:Ac ratios. Independent gel electrophoretic analysis was done three to four times/individual for the high-My:Ac and low-My:Ac groups, and usually two times/individual for the intermediate-My:Ac group; all data points are shown. $\mathbf{b}$ Western blot of $1.8 \%$ polyacrylamide gel loaded with biopsy samples of CIM muscles expressing high or low My:Ac ratios, using antibodies to pan-ubiquitin (pan-Ub). TPBS, total protein stain of blotted TTN proteins. c Western blot detecting expression of myomesin-1 (MYOM1) in CIM muscles with high or low My:Ac ratios. GAPDH, loading control. Blots shown in $\mathbf{b}$ and $\mathbf{c}$ are representative of three total blots performed. d Western blots detecting levels of heat shock proteins (HSP90, HSP70) and titin-binding mechanosensitive proteins (ANKRD1, FHL2) in CIM muscles expressing high to low My:Ac ratios. GAPDH, loading control. Right panels show results of densitometric analysis. Analysis was done on $n=4$ patients/group for HSP9O and HSP70; $n=3$ patients/group for ANKRD1, and $n=5$ patients/group for FHL2. e Representative electron micrographs (out of >25 images recorded per condition) of CIM muscles expressing high (left panel) and low My:Ac ratios (right panels). Note the pattern of sarcomere disassembly with preferential thick-filament depletion, loss of sarcomere orientation, and Z-disc protein aggregation. Bars, $1 \mu \mathrm{m}$ (black bars), and $0.5 \mu \mathrm{m}$ (white bars). Data in a and $\mathbf{d}$ are mean $\pm \mathrm{SD} .{ }^{\star \star \star} p<0.001,{ }^{\star \star} p<0.01,{ }^{\star} p<0.05, \mathrm{~ns}$ nonsignificant; unpaired, two-tailed Student's $t$-test with Welch's correction; for multiple comparisons, ANOVA followed by Sidak's test was also performed. Source data are provided as a Source Data file.

protein is characteristic of skeletal muscles from ventilated CIM patients in ICUs and that the TTN-loss accompanies the wellknown preferential loss of thick filaments in CIM. We propose that the sarcomere disruption and muscle wasting in peripheral skeletal muscles of CIM patients are, at least in part, due to mechanical silencing of titin.

Interestingly, our proof-of-concept homozygous TTN-KO mice failed to develop aligned cardiac sarcomeres. This finding is consistent with previous reports of genetically modified mice ${ }^{14}$ and human-induced pluripotent stem cell-derived cardiomyocytes (hiPSC-CMs) carrying TTN-truncating mutations, which suggested that contiguous TTN is critical for the initial stages of sarcomere assembly ${ }^{43}$. Further analysis of hiPSC-CMs with an Aband-TTN truncation implicated that intact TTN forms an essential mechanical link between sarcomeric myosin and the protocostamere-actinin (Z-body) complex as a prerequisite for sarcomere assembly ${ }^{13}$. Our constitutive TTN-KO model supports the view that full-length TTN provides a mechanical connection between the Z-bodies and the myosin filaments required for cardiac sarcomerogenesis. The A-band-bound Cronos isoform, which we recently found to support formation of sarcomeres in hiPSC-CMs ${ }^{31}$, does not rescue cardiac sarcomerogenesis in vivo in the absence of Z-disc-anchored titin.

Although TTN is widely thought to stabilize adult sarcomeres, direct proof has been missing. Our inducible, skeletal musclespecific TTN-KO mouse now unequivocally demonstrates that Zdisc-anchored TTN maintains the sarcomeric integrity in mature muscle cells. While this function is provided most likely by the half-sarcomere-spanning titin isoforms (such as N2A), it remains to be seen whether Novex-3, which was also deleted in our model, has an additional role in sarcomeric maintenance.

We found differential expression of Cronos in fast-type vs. fatigue-resistant murine skeletal muscles and exploited this fact to address the potential role of Cronos in rescuing the sarcomere structure $^{5}$ after depletion of Z-disc-anchored TTN. However, disintegration of the sarcomeres occurred independent of whether Cronos was present (M. gastrocnemius) or not (diaphragm), which excludes a role of Cronos in maintaining the structure of adult sarcomeres. Remarkably, there was a tendency for extended lifespan of remnant A-bands in muscles expressing Cronos. Overall, however, the protective effect of Cronos on the A-bands, if any, appeared to be rare and transient, and it did not prevent rapid detachment of Z-discs from A-bands upon TTN-KO. Generally, the changes in sarcomeric protein levels and ultrastructure with TTN-loss were similar in all muscle types studied. Thus, Cronos may play a role in stabilizing the A-band of healthy fast-type muscle sarcomeres, but it is unable to substitute for the function of full-length TTN in sarcomere proteostasis.

The role of TTN as a contributor to myocyte mechanical properties was confirmed here, as both passive stiffness (lateral and longitudinal) and active developed tension were reduced in dox-treated MUT vs. WT myofibers. An additional property of TTN is its mechanosensor function ${ }^{44-46}$. Mechanosensitive regions in titin's Z-, I- and M-band segments associate with molecules involved in hypertrophic/atrophic signaling, which are activated by stress or strain ${ }^{47}$. In the Z-disc, the TTN N-terminus interacts with the T-CAP/CSRP3 complex, a postulated key component of Z-disc-based mechanosignaling ${ }^{37}$. We found that TTN-loss in adult muscle profoundly increased T-CAP and CSRP3 expression. Previously, transgenic mice overexpressing TCAP were reported to develop less apoptosis than WT animals under elevated cardiac afterload, suggesting a role for the protein in regulated cell death ${ }^{48}$. CSRP3 is involved in myocyte autophagy, next to its roles in myocyte cytoarchitecture and myotube differentiation ${ }^{49}$. Considering these functions, the upregulation of T-CAP and CSRP3 in our MUT mouse may represent an adaptive mechanism to prevent muscle wasting-which however fails, as TTN becomes increasingly unable to provide mechanical support.

Other mechanical signaling molecules in myocytes binding to the N2A-region of I-band TTN are the MARPs ${ }^{40}$. Members of this protein family can modulate transcription factors and are deregulated under various muscle stresses ${ }^{50}$. The increase of Ankrd1 and Ankrd2 in our TTN-KO mice suggests deregulation of hypertrophy/atrophy signaling. Furthermore, the N2A-element and many Ig-domains in the TTN spring interact under stress with small HSPs, such as CRYAB ${ }^{51}$. Chaperones are important regulators of myocyte protein turnover and provide a first-line protective response to many types of stress ${ }^{52}$. At least part of the increase in CRYAB expression observed upon TTN-inactivation may be related to disrupted sHSP-titin interactions. The N2Aelement of TTN also associates with HSP90 in complex with cochaperone Smyd2; together, they protect the Z/I-band structure of the sarcomere ${ }^{53,54}$. HSP90 and another Smyd protein, Smyd1, also have crucial roles in chaperoning the myosin filament during sarcomere development and maintenance ${ }^{35,55}$. The abnormal expression of these (co)chaperones in our MUT muscles may indicate the (failed) attempt to protect sarcomeric proteins from pathological aggregation and excessive degradation.

Various other components of the cellular PQC machinery became activated as a consequence of the TTN-loss. The TTNbinding calpains, CAPN1 and CAPN $3^{56,57}$, were both elevated. 
Their increase suggests a role in sarcomeric protein degradation at early stages of myofibril breakdown ${ }^{33,58}$ in our MUT model. Furthermore, we observed substantially higher ubiquitination of TTN and other muscle proteins in dox-treated MUT vs. WT muscles as a sign of increased activity of the ubiquitinproteasome system and alternative pathways for targeted degradation ${ }^{30,52}$. This finding is consistent with previous studies in mouse models of muscle atrophy demonstrating that TTN becomes ubiquitinated at lysine residues mainly in the A-/Mband ${ }^{59,60}$. Interestingly, the muscle E3 ubiquitin ligases, Fbxo32 (atrogin-1) and Trim63 (MuRF1), were not significantly altered in our TTN-KO mice, although they are usually induced during muscle atrophy ${ }^{32}$. While TRIM63 binds directly to TTN at the M-band ${ }^{61}$, the E3 ligase has never been shown to ubiquitinate TTN; however, it does ubiquitinate many other sarcomere proteins, preferentially in thick filaments ${ }^{62}$. We speculate that other, yet-to-be-identified, E3 ligases may be activated by reduced supply of TTN, causing increased ubiquitination of remaining TTN protein. Moreover, two markers of autophagic-lysosomal activity, SQSTM1/p62 and LAMP2 ${ }^{63,64}$, were upregulated in MUT muscles. SQSTM1 binds via neighbor of BRCA1 gene-1 protein to the titin-kinase domain in M-band TTN ${ }^{36}$, thus linking stress-induced autophagic signaling to the sarcomere ${ }^{47}$. Although it is still unknown whether/how much TTN protein is degraded via autophagic pathways, the increase in autophagy markers upon TTN-KO suggests involvement of autophagic processes in the sarcomeric disintegration. Collectively, these results demonstrate that the TTN-loss increases the activity of all components of the PQC machinery, which jointly mediate the accelerated degradation of the myocyte proteins.

An intriguing finding of this study is the remarkable similarity in the pattern of sarcomere disintegration and myocyte protein changes between our TTN-KO mice and CIM patients presenting with peripheral muscle wasting. In TA muscles of CIM patients, progressive reduction in thick-filament proteins, such as MyHC, MyBPC and myomesin-1, was accompanied by a significant loss of TTN protein already at early stages of the muscle atrophy when the MyHC:actin expression ratio was only modestly reduced. Nebulin, actin, and $\alpha$-actinin levels showed little changes, whereas TTN-binding mechanosensitive proteins, chaperone HSP90, and the levels of ubiquitinated TTN increased progressively. A large body of evidence has demonstrated that CIM patient muscles upregulate, among others, components of the PQC machinery, including chaperones, proteases, and E3ubiquitin ligases ${ }^{65}$. Trim63 activation was speculated to cause the preferential myosin-loss in CIM patients ${ }^{26}$. Other proteins found to be elevated in CIM patient muscles are inflammatory molecules, transcriptional repressors, and mechanosensitive myocyte proteins ${ }^{65}$. We are not aware of any genetic animal model resembling the hallmarks of human CIM, i.e., the preferential loss of thick filaments and the pathophenotypic appearance of the myocyte/sarcomere ultrastructure, as closely as induced depletion of TTN.

In mechanically ventilated, deeply sedated and/or pharmacologically paralyzed ICU patients, there is a complete loss of mechanical stimuli of the skeletal muscles, including external strain (no weight bearing!) and internal strain (no activation of the contractile apparatus!), which presumably is an important trigger of this special myopathy. A sophisticated rat model has been developed to assist in defining the contributions of mechanical ventilation and muscle unloading to $\mathrm{CIM}^{66,67}$. Importantly, passive mechanical loading attenuated muscle wasting and myosin loss in this model ${ }^{68}$. Thus, mechanosensitive factors within the muscles are involved in the pathomechanisms of CIM. Our findings suggest the participation of TTN-based mechanosensing via TTN-binding signaling molecules.
Mechanical silencing of TTN due to muscle unloading may cause the loss of TTN in CIM, because mechanosensitive pathways normally triggered by TTN strain fail to be activated and regulate sarcomere protein turnover. Despite upregulation of TTNassociated factors, muscle wasting proceeds, and the pathologically lowered TTN levels cause sarcomeric disintegration. Presumably, loss of TTN precedes loss of myosin not only in our TTN-depletion mouse model but also in CIM. If this holds true, the TTN-KO mouse provides a new tool for studying the pathogenesis of CIM and may also help in the search for potential therapeutic approaches.

\section{Methods}

Animal experiments. All animal procedures were performed according to national guidelines (TierSchG and TierSchVersV) and were approved by local government authorities (Landesamt für Natur, Umwelt und Verbraucherschutz NordrheinWestfalen, LANUV, Az 84-02.04.2015.A261).

Animal models. The titin knockout-first mice were generated by injecting EPD0145_2_G01 ES cell clones from the EUCOMM (European Conditional Mouse Mutagenesis Program) Consortium into BAlb/C blastocysts. The specifics and the complete DNA sequence of the Ttn gene targeting construct are available in the GenBank ${ }^{\circledast}$ database (accession no. JN959336.1). Validation of inserted cassette integrity and correct targeting are described in supplementary methods (Supplementary Fig. 1). The chimeric mice obtained were mated to C57BL/6JRj mice (Janvier Labs, France) to generate heterozygous knockout-first mice $\left(\operatorname{Ttn}^{\mathrm{tmla} /+}\right)$ and to Flipase (Flp)-expressing transgenic mice (Flp-Deleter) ${ }^{69}$ to generate the lacZ-neo cassette-deleted, heterozygous, conditional Ttn $\mathrm{KO}$ mice $\left(\operatorname{Ttn}^{\mathrm{tm} l \mathrm{c}} /+\right)$.

The inducible skeletal muscle-specific Ttn $\mathrm{KO}$ mice were generated by crossing the conditional Ttn KO mice ( $\mathrm{Ttn}^{\mathrm{tm} l \mathrm{c} /+}$ or $\left.\mathrm{Ttn}{ }^{\mathrm{tm} 1 \mathrm{c} / \mathrm{tm} l \mathrm{c}}\right)$ to the B6;C3-Tg(ACTA1rtTA,tetO-cre) ${ }^{102 M o n k / J}$ mouse line, purchased from the Jackson Laboratory (Bar Harbor, Maine, USA), which expresses Cre-recombinase under the control of the tetracycline-responsive regulatory element (tetO/TRE) and a reverse tetracyclinecontrolled transactivator (rtTA) under the control of the human actin, alpha 1 skeletal muscle (ACTA1) promoter ${ }^{28}$

As a reporter line for the effectiveness of the dox-treatment, we used a Rosa26$\mathrm{LacZ} / \mathrm{Tg}$ (ACTA1-rtTA,tetO-cre) hybrid line, generated by mating the B6;C3-Tg (ACTA1-rtTA,tetO-cre) ${ }^{102 M o n k / J}$ mouse line to the B6;129S4-Gt(ROSA)

26 Sor $^{\text {tm1Sor/J strain. The latter is the R26R Gtrosa26 targeted mutant mouse }}{ }^{70}$ carrying lacZ flanked by loxP sites (Supplementary Fig. 4).

Primers used for genotyping are shown in Supplementary Table 1.

Transgene induction. In 8-30 week-old $\mathrm{Ttn}^{\text {tmlc/tmlc; }}$;CTA1-rtTApos;tetO-crepos and gender-matched WT littermates, Cre-mediated recombination was achieved by administering the tetracycline analog doxycycline. Doxycycline hydrochloride (dox; Sigma, D9891) was dissolved in drinking water at a concentration of $2 \mathrm{mg} / \mathrm{mL}$ supplemented with $5 \%$ sucrose and was provided ad libitum in light-proofed water bottles for 27-38 days. Treatment was terminated when mice had reduced their body weight by $10-20 \%$. Tissues were then collected from mutant and WT mice for further experiments.

Morphometric and histological analyses. Mice were sacrificed by cervical dislocation and embryos, adult hearts and skeletal muscles were dissected. Body weight was recorded and muscle weights measured relative to tibia length. Tissues were fixed with $4 \%$ paraformaldehyde (PFA) in phosphate-buffered saline (PBS) for paraffin- or cryo-embedding, or with Zamboni's fixative for electron microscopy, and placed on a shaking table at $4{ }^{\circ} \mathrm{C}$ overnight. Cryosections of $\mathrm{M}$. triceps brachii were stained with hematoxylin or hematoxylin/eosin to observe differences between dox-treated WT and MUT. Digital images of hematoxylin/eosin-stained cryosections of triceps brachii were processed and analyzed using ImageJ software (NIH, Bethesda, USA), to measure cell number, fiber cross-sectional area, and number of nuclei. The percentage of centrally nucleated fibers was determined for at least 225 (up to 488) fibers per muscle, using photomicrographs taken at two different positions along the muscle. The cross-sectional area was determined on at least 160 fibers per animal at different positions along the entire muscle.

Muscle physiology assessment. The $4 \mathrm{LHT}$ or inverted screen test ${ }^{71}$ was used to

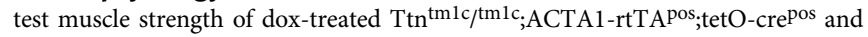
WT mice. Briefly, mice were placed on a wire mesh consisting of $6 \mathrm{~mm}$ squares of $1 \mathrm{~mm}$ diameter wire, surrounded by $5 \mathrm{~cm}$ edging to prevent climbing on the other side. Immediately after placing the mouse in the center, the screen was turned upside down, fixed in this position $40-50 \mathrm{~cm}$ above a soft-bedded cage, and the hang time was measured. The test ended when a hang time of $15 \mathrm{~min}$ was reached; if not, the test ended after two sessions. The maximum hang time was recorded. 
In situ hybridizations. Following overnight-fixation in $4 \%$ PFA, tissues were dehydrated in a graded ethanol series, paraffin-embedded, cut into $8 \mu \mathrm{m}$ sections, and mounted on Superfrost ${ }^{\text {tx }}$ Plus microscope slides. The Ttn exon 20_24 probe was derived from Ttn N2A sequence positions 3372-3483 (reference sequence: NM_011652.3, primer sequences in Supplementary Table 1) and digoxigeninlabeled according to the manufacturer's specifications (Roche, Basel). In situ hybridizations on sections were performed ${ }^{72}$ with the following modifications: sections were pre-hybridized in hybridization mix without probe for $1-2 \mathrm{~h}$ at $60^{\circ} \mathrm{C}$ and then hybridized overnight at $60^{\circ} \mathrm{C}$. Approximately $250 \mu \mathrm{L}$ of hybridization mix were applied to the slide and the sections covered by a coverslip. Probe concentration was $\sim 1 \mathrm{ng} / \mu \mathrm{L}$.

RT-PCR and quantitative real-time PCR analysis. Total RNA was prepared from tissues using Trizol $^{\oplus}$ (Invitrogen) according to the manufacturer's protocol. RNA quality and quantity were estimated by comparing the fluorescence signals of the $28 \mathrm{~S}$ and $18 \mathrm{~S}$ rRNA bands on agarose gels. Reverse transcription was carried out using Oligo(dT) and Random primers, and RNA polymerase M-MLV RT (Promega, Madison, USA) according to the manufacturer's instructions. Transcriptspecific primers are reported in Supplementary Table 1.

Quantitative real-time PCR (qPCR) analysis was performed on samples obtained from at least 3 animals per genotype and used for quantification of relative gene-expression data using the RQ $\left(2^{-\Delta \Delta C T}\right)$. The hypoxanthine-guanine phosphoribosyl transferase gene (Hprt; accession, NM_013556) served as the reference gene, unless otherwise stated. The qPCR was performed on the StepOne ${ }^{\mathrm{wx}}$ Real-Time PCR System (Applied Biosystems) using the following 2-step program: initial denaturation at $95^{\circ} \mathrm{C}$ for $10 \mathrm{~min}$ followed by 40 cycles of denaturation for $15 \mathrm{~s}$ at $95^{\circ} \mathrm{C}$ and annealing/extension for $1 \mathrm{~min}$ at $60^{\circ} \mathrm{C}$, terminated by a final denaturation step at $95^{\circ} \mathrm{C}$ for $15 \mathrm{~s}$. For qRT-PCR reactions, the GoTaq qPCR Master Mix reagent was used. Amplicon specificity was confirmed by examination of the dissociation curves. A distinct single peak indicated that a specific DNA sequence had been amplified.

SDS-PAGE and western blot analysis. Snap-frozen heart and skeletal muscle tissues were solubilized in titin sample buffer ( $8 \mathrm{M}$ urea, $2 \mathrm{M}$ thiourea, 3\% SDS, $0.05 \mathrm{M}$ Tris, $10 \%$ glycerol, $75 \mathrm{mM}$ DTT, $0.03 \%$ Serva Blue) by cutting them in very small pieces and incubating them for $20 \mathrm{~min}$ on ice. After boiling the probes for 3 min at $96^{\circ} \mathrm{C}$, the debris was spun down for 5 min at $20,000 \times g$ and directly loaded on gels or frozen at $-80^{\circ} \mathrm{C}$ until use.

The concentration of total protein in each sample was assessed using a Bradford reagent (Sigma). For the separation of giant myofibrillar proteins, protein lysates $(7-30 \mu \mathrm{g})$ were resolved overnight in agarose-stabilized $1.8-3 \%$ slab gels $^{23,34}$. Proteins up to $250 \mathrm{kDa}$ were resolved by standard SDS-PAGE $(15 \%, 12.5 \%, 10 \%$, or $8 \%$ ). For simultaneous separation of TTN and proteins up to $250 \mathrm{kDa}$, we combined both methods, using for the stacking gel an agarose-stabilized 1.8-2.5\% gel and for the running gel the standard SDS-PAGE recipe $(12.5 \%, 10 \%, 8 \%, 6 \%)$. Electrophoresis was performed at $2 \mathrm{~mA} /$ plate at room temperature overnight. After separation, proteins were transferred onto PVDF membranes (Millipore) by using the Trans-Blot ${ }^{\circledast}$ Turbo $^{\text {nx }}$ transfer system (Bio-Rad). After transfer, total protein blot stain (TPBS) on the membranes was performed with Coomassie brilliant blue (1610436, Sigma-Aldrich). Myosin or actin bands were used as an additional loading control.

Detailed information on the primary and secondary antibodies is provided in Supplementary Table 2. Validation of custom-made antibodies is described in Supplementary Methods and results are shown in Supplementary Fig. 9. Signals from HRP-conjugated secondary antibodies were visualized by using chemiluminescence (Amersham ECL start Western Blotting Detection Reagent, GE Healthcare) and recorded using the ImageQuant LAS 4000 Imaging System (GE Healthcare). Signal intensity was quantified using the ImageQuant TL software (GE Healthcare).

Immunohistochemistry. Muscle tissues were prepared for immunohistochemistry using standard protocols. Briefly, samples were fixed with $4 \%$ formaldehyde solution in PBS and embedded in paraffin; $10-\mu \mathrm{m}$-thick sections were prepared. After deparaffinization and rehydration, antigen unmasking was achieved by shortly boiling the slides, submersed in $1 \mathrm{mM}$ EDTA ( $\mathrm{pH} 8)$, using a microwave oven. After incubation for an additional $20 \mathrm{~min}$ at a sub-boiling temperature, slides were washed in distilled water and incubated in blocking buffer $(0.1 \%$ Triton X-100 and $10 \%$ goat serum in PBS) for $1 \mathrm{~h}$. Antibodies were diluted in staining buffer ( $0.1 \%$ bovine serum albumin in PBS). The primary antibodies were applied overnight at $4{ }^{\circ} \mathrm{C}$, the secondary antibodies for $2 \mathrm{~h}$ at room temperature, with three washes in between incubations. Detailed information on the primary and secondary antibodies is given in Supplementary Table 2. Sections were embedded in Mowiol and images were recorded at room temperature using a confocal laser scanning system (Nikon A1) equipped with an Eclipse Ti inverted microscope.

Quantification of cross striations. Immunofluorescence staining on paraffin sections was performed using anti-titin Z-disc (TTN Z/I-2080) and anti- $\alpha$-actinin antibodies (Supplementary Table 2) to determine the number of cells with or without cross striations in WT and MUT muscles $(n=3$ gastrocnemius muscles per group) after dox-treatment for 21 or $\sim 30$ days. Images were recorded using a Nikon Al confocal microscope. The striation pattern of at least 90 muscle fibers per animal was evaluated.

Cryo embedding. After incubation in $4 \%$ PFA at $4{ }^{\circ} \mathrm{C}$ overnight, muscle tissues were placed in $15 \%$ sucrose in PBS, then in 30\% sucrose in PBS and slightly agitated at $4{ }^{\circ} \mathrm{C}$, until the tissue had receded. Afterward, tissues were embedded in optimal cutting temperature compound and cooled down on dry ice. Cryosectioning was performed on a Leica CM300 Cryostat.

Electron microscopy and immunoelectron microscopy. Mouse skeletal muscle and CIM patient biopsy samples were fixed in $4 \%$ PFA, $15 \%$ saturated picric acid in $0.1 \mathrm{M} \mathrm{PBS}, \mathrm{pH} 7.4$, at $4{ }^{\circ} \mathrm{C}$ overnight. For ultrastructural analysis without antibody labeling, tissue samples were embedded in resin and processed for transmissionEM. For immuno-EM, longitudinal sections were cut on a vibratome (Leica VT $1000 \mathrm{~S}$ ) at a thickness of $50 \mu \mathrm{m}$, blocked in $20 \%$ normal goat serum (NGS, Vector Laboratories, Burlingame, USA) in PBS and were incubated with primary antibodies diluted in PBS containing 5\% NGS overnight at $4{ }^{\circ} \mathrm{C}$. Antibody references and corresponding dilutions are provided in Supplementary Table 2. After several washes in PBS, the sections were incubated with $1.4 \mathrm{~nm}$ gold-coupled secondary antibodies (Nanoprobes, Yaphank, NY, USA) in PBS at $4{ }^{\circ} \mathrm{C}$ overnight. After extensive washing, the sections were postfixed in $1 \%$ glutaraldehyde in PBS for 10 min and then reacted with HQ Silver kit (Nanoprobes). Following treatment with osmium tetroxide, sections were stained with uranyl acetate, dehydrated, and embedded in Durcupan resin (Fluka, Switzerland). Ultrathin sections were prepared (Ultracut S; Leica, Germany) and adsorbed onto glow-discharged Formvarcarbon-coated copper grids. Microscopy was performed on a Zeiss LEO 910 electron microscope and images were taken with a TRS sharpeye CCD camera (TRS Systems, Moorenweis, Germany).

Quantification of Z-disc perimeter. On electron micrographs of WT and KO (10-20\% BWL) ultrathin sections of gastrocnemius muscle tissue $(N=2$ animals, $250 \mathrm{Z}$-discs/group), we calculated the Z-disc circumference by using ImageSP software (TRS Systems, Moorenweis, Germany). Z-disc boundaries were clearly identified by their electron densities and perimeters were obtained via ImageSP polygon measurement. Full image information was transferred to Microsoft Excel for statistical analysis.

AFM-based myofiber stiffness measurements. To determine the mechanical stiffness of WT and MUT skeletal muscle cells, single isolated fibers were probed by nanoindentation measurements using an atomic force microscope (Nanowizard 3 AFM system, JPK, Germany). Deep-frozen tissue was defrosted and skinned overnight in ice-cold low ionic-strength buffer $(75 \mathrm{mM} \mathrm{KCl}, 10 \mathrm{mM}$ Tris, $2 \mathrm{mM}$ $\mathrm{MgCl}_{2}, 2 \mathrm{mM}$ EGTA, and $40 \mu \mathrm{g} / \mathrm{mL}$ protease inhibitor leupeptin, $\mathrm{pH}$ 7.2) supplemented with $0.5 \%$ Triton X-100. Under a binocular (Leica, Mannheim, Germany), single myofibers were manually dissected and placed in relaxing buffer (8 mM ATP, $20 \mathrm{mM}$ imidazole, $4 \mathrm{mM}$ EGTA, $12 \mathrm{mM}$ Mg-propionate, $97 \mathrm{mM}$ K-propionate, $\mathrm{pH}$ 7.2) at room temperature. The nanoindentation technique was applied as follows: dissected myofibers in relaxing buffer were placed in a fluid chamber, in which the cover glass had been coated with the glue Cell-Tak (DLW354240, Sigma-Aldrich, Germany); fibers thus adhered tightly to the cover glass as a prerequisite for reliable force-indentation measurements. A precalibrated cantilever (spring constant, $k_{\mathrm{C}}=0.03-0.04 \mathrm{~N} \mathrm{~m}^{-1}$, Novascan Technologies, USA) with a mounted spherical polystyrene bead (diameter, $10 \mu \mathrm{m}$ ) periodically indented the myofiber up to a pre-set indentation force of $3 \mathrm{nN}$. During the measurements, the AFM tip was moved vertically toward the sample surface at constant velocity of $2 \mu \mathrm{m} \mathrm{s}^{-1}$. Upon indentation of the myofiber, the AFM cantilever became deflected. The relative position of a laser beam, focused on the cantilever tip and reflected back to a photodiode detector, was used to quantify cantilever deflection and generate force-vs.-indentation curves. From the force acting on the cantilever, the piezo displacement, and the deflection sensitivity, the indentation depth was calculated. By subtracting the deflection of the cantilever from the piezo movement after contacting the myofiber, the force-distance curves were converted to force-indentation curves. The slope of the force-indentation curve indicated the lateral stiffness of the myofiber. The Young's modulus was obtained by fitting the force-indentation curve from the contact point to the end (maximum indentation) using the Hertz model corrected for a spherical indenter (JPK data processing software, JPK, Germany), according to the equation:

$$
F=\frac{4 E \cdot R^{\frac{1}{2}}}{3\left(1-v^{2}\right)} \cdot \delta^{\frac{3}{2}}
$$

where $F$ is the indentation force, $E$ is the Young's modulus, $R$ is the radius of the sphere directly contacting the sample surface $(R=5 \mu \mathrm{m}$ in the current experiments), $v$ is the Poisson's ratio of the sample $(v=0.5$, commonly used for biological samples), and $\delta$ is the measured indentation depth.

Passive and active tension measurements on single myofibers. Dissected vastus lateralis muscle from $\sim 30$-day dox-treated WT and MUT mice was skinned 
overnight in ice-cold relaxing buffer (2.5 mM ATP, $14.5 \mathrm{mM}$ phosphocreatine, $20 \mathrm{mM}$ MOPS, $5 \mathrm{mM} \mathrm{K} \mathrm{K}_{2}$ EGTA, $2.5 \mathrm{mM}$ MgAcetate, $170 \mathrm{mM}$ K-propionate, $\mathrm{pH} 7.2$ ) supplemented with $0.5 \%$ Triton X-100 and protease inhibitor cocktail (G6521, Promega). After washing three times with relaxing buffer, myofiber isolation was carefully done on ice, avoiding excessive stretching. Single myofibers were attached between a motor and a force transducer (Scientific Instruments, Heidelberg, Germany) and mechanical experiments run at room temperature. Fiber cross-sectional area was estimated from the fiber diameter (assuming a circular shape) measured at slack length. For passive tension measurements, fibers were stretched from slack length in six equidistant steps (maximum strain, 1.55). After each step, force relaxation was allowed for during a 1-min hold period. The peak force value immediately after each stretch was converted to stress (force per cross-sectional area). For active tension measurements, fibers were pre-stretched (strain, 1.2) in relaxing buffer for $2 \mathrm{~min}$ and then transferred to activating solution (pCa 4.5; $2.5 \mathrm{mM}$ ATP, $10 \mathrm{mM}$ MOPS, $5 \mathrm{mM}$ CaEGTA, $2.4 \mathrm{mM}$ Mg-Acetate, $170 \mathrm{mM} \mathrm{K}$ propionate, $\mathrm{pH}$ 7.2), while force was recorded. Maximum active force was normalized to fiber cross-sectional area $\left(\mathrm{Ca}^{2+}\right.$-activated tension). All solutions used for mechanical experiments contained protease inhibitor cocktail (G6521, Promega).

Biopsies of CIM patient muscles. Percutaneous conchotome muscle biopsies were taken from the TA muscle of 9 mechanically ventilated ICU patients with CIM (intermediate myosin:actin ratios: 3 women and 2 men, 62-79 years; low myosin:actin ratios: 3 women and 1 man, $41-79$ years) and 5 control immobilized ICU patients with lesions in the central or peripheral nervous system ( 2 women and 3 men, 56-70 years). All ICU patients with CIM had been mechanically ventilated for longer than two weeks at the time of muscle biopsy. The biopsies are from patients recruited in a study aiming at improving the diagnostic precision of CIM, i.e., comparing results from percutaneous muscle biopsies with results from biopsies obtained with a disposable microbiopsy instrument. Thus, ICU patients were included who had developed a general paralysis in response to long-term mechanical ventilation and were referred to the clinic for diagnostic purposes. Control samples displaying high myosin:actin ratios were obtained on the first day on the ventilator prior to any myosin loss. In these patients, a myosin loss was evident first after more than one week of immobilization and mechanical ventilation. There is no indication that the inclusion of these patients may have introduced any bias which may have impacted on the results. Each biopsy was frozen in liquid propane chilled by liquid nitrogen and stored at $-140^{\circ} \mathrm{C}$ or -160 ${ }^{\circ} \mathrm{C}$ until analyses. Written informed consent was obtained from patients' close relatives prior to the study. The study was approved by the Ethical Committee on Human Research at Karolinska Institutet, Stockholm and Uppsala University Hospital, Sweden (approval number, 2016/242-31/2). Informed consent was obtained from all patients or close relatives (deeply sedated and mechanically ventilated ICU patients).

Statistical analysis. Data organization, scientific graphing and statistical analyses were performed using Microsoft Excel (2013) and GraphPad Prism (version 7). Results are presented as the mean $\pm S D$, mean \pm SEM, or as the difference between means \pm SEM, as indicated. Normality was assessed using the Shapiro-Wilk test. Significant differences between two groups were determined by two-tailed Student's $t$-test or Mann-Whitney test and differences with p-values lower than 0.05 were considered significant. Variance analyses of repeated measurements were performed by repeated measures two-way ANOVA, followed by Sidak's test for multiple comparisons; here, differences with $p$ values lower than 0.01 were considered significant. Sample size was estimated based on sample availability, previous experimental studies performed in our laboratory, and the literature. Experiments were not randomized or blinded.

Reporting summary. Further information on research design is available in the Nature Research Reporting Summary linked to this article.

\section{Data availability}

All data pertaining to this work are shown in the text, figures, and Supplementary Information. The source data underlying Figs. 1e, 2a, b, d, e, 3c, g, 4b-d, 5a-d, 6b, d, $7 \mathrm{~b}-\mathrm{d}, 8 \mathrm{a}, \mathrm{d}$, and supplementary figs. $2 \mathrm{e}, 5 \mathrm{~b}, 6 \mathrm{~b}-\mathrm{d}$, and 7 are provided as a Source Data file. Full scans of images shown in panels $1 c, d, 5 b, d, 6 a, c, 7 a, c, d, 8 b-d$, and supplementary figs. $2 \mathrm{~b}, \mathrm{~d}, 6 \mathrm{c}, 8 \mathrm{a}, \mathrm{b}$, and $9 \mathrm{e}$ are also provided as a separate Source Data file. Source data are provided with this paper.

Received: 29 June 2019; Accepted: 4 August 2020;

Published online: 08 September 2020

\section{References}

1. Furst, D. O., Osborn, M., Nave, R. \& Weber, K. The organization of titin filaments in the half-sarcomere revealed by monoclonal antibodies in immunoelectron microscopy: a map of ten nonrepetitive epitopes starting at the $\mathrm{Z}$ line extends close to the M line. J. Cell Biol. 106, 1563-1572 (1988).
2. Gregorio, C. C. et al. The NH2 terminus of titin spans the Z-disc: its interaction with a novel $19-\mathrm{kD}$ ligand (T-cap) is required for sarcomeric integrity. J. Cell Biol. 143, 1013-1027 (1998).

3. Bang, M. L. et al. The complete gene sequence of titin, expression of an unusual approximately $700-\mathrm{kDa}$ titin isoform, and its interaction with obscurin identify a novel Z-line to I-band linking system. Circ. Res. 89, 1065-1072 (2001).

4. Savarese, M. et al. The complexity of titin splicing pattern in human adult skeletal muscles. Skelet. Muscle 8, 11 (2018).

5. Zou, J. et al. An internal promoter underlies the difference in disease severity between N- and C-terminal truncation mutations of Titin in zebrafish. Elife 4, e09406 (2015).

6. Wang, K. Sarcomere-associated cytoskeletal lattices in striated muscle. Review and hypothesis. Cell Muscle Motil. 6, 315-369 (1985).

7. Maruyama, K., Matsubara, S., Natori, R., Nonomura, Y. \& Kimura, S. Connectin, an elastic protein of muscle. Characterization and Function. J. Biochem. 82, 317-337 (1977).

8. Granzier, H. \& Labeit, S. Cardiac titin: an adjustable multi-functional spring. $J$. Physiol. 541, 335-342 (2002).

9. Linke, W. A. \& Fernandez, J. M. Cardiac titin: molecular basis of elasticity and cellular contribution to elastic and viscous stiffness components in myocardium. J. Muscle Res. Cell Motil. 23, 483-497 (2002).

10. Rivas-Pardo, J. A. et al. Work done by titin protein folding assists muscle contraction. Cell Rep. 14, 1339-1347 (2016).

11. Linke, W. A. Titin gene and protein functions in passive and active muscle. Annu Rev. Physiol. 80, 389-411 (2018).

12. Tonino, P. et al. The giant protein titin regulates the length of the striated muscle thick filament. Nat. Commun. 8, 1041 (2017).

13. Chopra, A. et al. Force generation via beta-cardiac myosin, titin, and alphaactinin drives cardiac sarcomere assembly from cell-matrix adhesions. Dev. Cell 44(87-96), e5 (2018).

14. Gramlich, M. et al. Stress-induced dilated cardiomyopathy in a knock-in mouse model mimicking human titin-based disease. J. Mol. Cell Cardiol. 47, 352-358 (2009).

15. Weinert, S., Bergmann, N., Luo, X., Erdmann, B. \& Gotthardt, M. M linedeficient titin causes cardiac lethality through impaired maturation of the sarcomere. J. Cell Biol. 173, 559-570 (2006).

16. Gotthardt, $M$. et al. Conditional expression of mutant $M$-line titins results in cardiomyopathy with altered sarcomere structure. J. Biol. Chem. 278, 6059-6065 (2003).

17. Person, V., Kostin, S., Suzuki, K., Labeit, S. \& Schaper, J. Antisense oligonucleotide experiments elucidate the essential role of titin in sarcomerogenesis in adult rat cardiomyocytes in long-term culture. J. Cell Sci. 113(Pt 21), 3851-3859 (2000).

18. Schafer, S. et al. Titin-truncating variants affect heart function in disease cohorts and the general population. Nat. Genet. 49, 46-53 (2017).

19. Radke, M. H. et al. Targeted deletion of titin N2B region leads to diastolic dysfunction and cardiac atrophy. Proc. Natl Acad. Sci. USA 104, 3444-3449 (2007).

20. Buck, D. et al. Removal of immunoglobulin-like domains from titin's spring segment alters titin splicing in mouse skeletal muscle and causes myopathy. J. Gen. Physiol. 143, 215-230 (2014).

21. Chung, C. S. et al. Shortening of the elastic tandem immunoglobulin segment of titin leads to diastolic dysfunction. Circulation 128, 19-28 (2013).

22. Granzier, H. L. et al. Truncation of titin's elastic PEVK region leads to cardiomyopathy with diastolic dysfunction. Circ. Res. 105, 557-564 (2009).

23. Brynnel, A. et al. Downsizing the molecular spring of the giant protein titin reveals that skeletal muscle titin determines passive stiffness and drives longitudinal hypertrophy. Elife 7, e40532 (2018).

24. Charton, K. et al. Removal of the calpain 3 protease reverses the myopathology in a mouse model for titinopathies. Hum. Mol. Genet. 19, 4608-4624 (2010).

25. Radke, M. H. et al. Deleting full length titin versus the titin m-band region leads to differential mechanosignaling and cardiac phenotypes. Circulation 139, 1813-1827 (2019).

26. Friedrich, O., Diermeier, S. \& Larsson, L. Weak by the machines: muscle motor protein dysfunction - a side effect of intensive care unit treatment. Acta Physiol. (Oxf) 222, e12885 (2018).

27. Larsson, L. et al. Acute quadriplegia and loss of muscle myosin in patients treated with nondepolarizing neuromuscular blocking agents and corticosteroids: mechanisms at the cellular and molecular levels. Crit. Care Med. 28, 34-45 (2000).

28. Rao, P. \& Monks, D. A. A tetracycline-inducible and skeletal muscle-specific Cre recombinase transgenic mouse. Dev. Neurobiol. 69, 401-406 (2009).

29. Talmadge, R. J. Myosin heavy chain isoform expression following reduced neuromuscular activity: potential regulatory mechanisms. Muscle Nerve 23, 661-679 (2000)

30. Kwon, Y. T. \& Ciechanover, A. The ubiquitin code in the ubiquitinproteasome system and autophagy. Trends Biochem. Sci. 42, 873-886 (2017), 
31. Zaunbrecher, R. J. et al. Cronos titin is expressed in human cardiomyocytes and necessary for normal sarcomere function. Circulation 140, 1647-1660 (2019).

32. Bodine, S. C. \& Baehr, L. M. Skeletal muscle atrophy and the E3 ubiquitin ligases MuRF1 and MAFbx/atrogin-1. Am. J. Physiol. Endocrinol. Metab. 307, E469-E484 (2014).

33. Portbury, A. L., Willis, M. S. \& Patterson, C. Tearin' up my heart: proteolysis in the cardiac sarcomere. J. Biol. Chem. 286, 9929-9934 (2011).

34. Anderson, L. V. et al. Characterization of monoclonal antibodies to calpain 3 and protein expression in muscle from patients with limb-girdle muscular dystrophy type 2A. Am. J. Pathol. 153, 1169-1179 (1998).

35. Just, S. et al. The myosin-interacting protein SMYD1 is essential for sarcomere organization. J. Cell Sci. 124, 3127-3136 (2011).

36. Lange, $\mathrm{S}$. et al. The kinase domain of titin controls muscle gene expression and protein turnover. Science 308, 1599-1603 (2005).

37. Knoll, R. et al. The cardiac mechanical stretch sensor machinery involves a $Z$ disc complex that is defective in a subset of human dilated cardiomyopathy. Cell 111, 943-955 (2002).

38. Sheikh, F. et al. An FHL1-containing complex within the cardiomyocyte sarcomere mediates hypertrophic biomechanical stress responses in mice. $J$. Clin. Invest. 118, 3870-3880 (2008).

39. Lange, S. et al. Subcellular targeting of metabolic enzymes to titin in heart muscle may be mediated by DRAL/FHL-2. J. Cell Sci. 115, 4925-4936 (2002).

40. Miller, M. K. et al. The muscle ankyrin repeat proteins: CARP, ankrd2/Arpp and DARP as a family of titin filament-based stress response molecules. J. Mol. Biol. 333, 951-964 (2003).

41. Lange, S. et al. MLP and CARP are linked to chronic PKCalpha signalling in dilated cardiomyopathy. Nat. Commun. 7, 12120 (2016).

42. Fornasiero, E. F. et al. Precisely measured protein lifetimes in the mouse brain reveal differences across tissues and subcellular fractions. Nat. Commun. 9 , 4230 (2018).

43. Hinson, J. T. et al. HEART DISEASE. Titin mutations in iPS cells define sarcomere insufficiency as a cause of dilated cardiomyopathy. Science 349, 982-986 (2015).

44. Ottenheijm, C. A., van Hees, H. W., Heunks, L. M. \& Granzier, H. Titin-based mechanosensing and signaling: role in diaphragm atrophy during unloading? Am. J. Physiol. Lung Cell Mol. Physiol. 300, L161-L166 (2011).

45. Gautel, M. Cytoskeletal protein kinases: titin and its relations in mechanosensing. Pflug. Arch. 462, 119-134 (2011).

46. Linke, W. A. Sense and stretchability: the role of titin and titin-associated proteins in myocardial stress-sensing and mechanical dysfunction. Cardiovasc Res. 77, 637-648 (2008).

47. Braun, T. \& Gautel, M. Transcriptional mechanisms regulating skeletal muscle differentiation, growth and homeostasis. Nat. Rev. Mol. Cell Biol. 12, 349-361 (2011).

48. Knoll, R. et al. Telethonin deficiency is associated with maladaptation to biomechanical stress in the mammalian heart. Circ. Res. 109, 758-769 (2011).

49. Vafiadaki, E., Arvanitis, D. A., Sanoudou, D. \& Muscle, L. I. M. Protein: master regulator of cardiac and skeletal muscle functions. Gene 566, 1-7 (2015).

50. Lun, A. S., Chen, J. \& Lange, S. Probing muscle ankyrin-repeat protein (MARP) structure and function. Anat. Rec. (Hoboken) 297, 1615-1629 (2014).

51. Kotter, S. et al. Human myocytes are protected from titin aggregationinduced stiffening by small heat shock proteins. J. Cell Biol. 204, 187-202 (2014).

52. Carlisle, C., Prill, K. \& Pilgrim, D. Chaperones and the proteasome system: regulating the construction and demolition of striated muscle. Int. J. Mol. Sci. 19 (2017).

53. Donlin, L. T. et al. Smyd2 controls cytoplasmic lysine methylation of Hsp90 and myofilament organization. Genes Dev. 26, 114-119 (2012).

54. Munkanatta Godage, D. N. P. et al. SMYD2 glutathionylation contributes to degradation of sarcomeric proteins. Nat. Commun. 9, 4341 (2018).

55. Hawkins, T. A. et al. The ATPase-dependent chaperoning activity of Hsp90a regulates thick filament formation and integration during skeletal muscle myofibrillogenesis. Development 135, 1147-1156 (2008).

56. Raynaud, F. et al. Calpain 1-titin interactions concentrate calpain 1 in the Zband edges and in the N2-line region within the skeletal myofibril. FEBS J. 272, 2578-2590 (2005).

57. Sorimachi, H. et al. Muscle-specific calpain, p94, responsible for limb girdle muscular dystrophy type $2 \mathrm{~A}$, associates with connectin through IS2, a p94specific sequence. J. Biol. Chem. 270, 31158-31162 (1995).

58. Huang, J. \& Zhu, X. The molecular mechanisms of calpains action on skeletal muscle atrophy. Physiol. Res. 65, 547-560 (2016).

59. Lang, F. et al. Dynamic changes in the mouse skeletal muscle proteome during denervation-induced atrophy. Dis. Model Mech. 10, 881-896 (2017).
60. Ryder, D. J. et al. Identification of the acetylation and ubiquitin-modified proteome during the progression of skeletal muscle atrophy. PLOS ONE 10, e0136247 (2015).

61. Centner, T. et al. Identification of muscle specific ring finger proteins as potential regulators of the titin kinase domain. J. Mol. Biol. 306, 717-726 (2001).

62. Cohen, S. et al. During muscle atrophy, thick, but not thin, filament components are degraded by MuRF1-dependent ubiquitylation. J. Cell Biol. 185, 1083-1095 (2009).

63. Mizushima, N. Autophagy: process and function. Genes Dev. 21, 2861-2873 (2007).

64. Nishino, I. et al. Primary LAMP-2 deficiency causes X-linked vacuolar cardiomyopathy and myopathy (Danon disease). Nature 406, 906-910 (2000).

65. Friedrich, O. et al. The Sick and the Weak: Neuropathies/Myopathies in the Critically Ill. Physiol. Rev. 95, 1025-1109 (2015).

66. Ochala, J. et al. Preferential skeletal muscle myosin loss in response to mechanical silencing in a novel rat intensive care unit model: underlying mechanisms. J. Physiol. 589, 2007-2026 (2011).

67. Norman, H. et al. Impact of post-synaptic block of neuromuscular transmission, muscle unloading and mechanical ventilation on skeletal muscle protein and mRNA expression. Pflug. Arch. 453, 53-66 (2006).

68. Renaud, G. et al. Sparing of muscle mass and function by passive loading in an experimental intensive care unit model. J. Physiol. 591, 1385-1402 (2013)

69. Rodriguez, C. I. et al. High-efficiency deleter mice show that FLPe is an alternative to Cre-loxP. Nat. Genet. 25, 139-140 (2000)

70. Soriano, P. Generalized lacZ expression with the ROSA26 Cre reporter strain. Nat. Genet. 21, 70-71 (1999).

71. Carlson, C. G. et al. A simple protocol for assessing inter-trial and interexaminer reliability for two noninvasive measures of limb muscle strength. $J$. Neurosci. Methods 186, 226-230 (2010).

72. Moorman, A. F., Houweling, A. C., de Boer, P. A. \& Christoffels, V. M. Sensitive nonradioactive detection of mRNA in tissue sections: novel application of the whole-mount in situ hybridization protocol. J. Histochem. Cytochem. 49, 1-8 (2001)

\section{Acknowledgements}

We thank Susanne Kreutzer (Bad Nauheim) for blastocyst injection, Sonja Krüger (Bad Nauheim) for ES cell propagation, Michael Yekelchyk (Bad Nauheim) for sharing expression data of muscle satellite cells, Franziska Koser (Münster) for help with titin gels, and Lisa Kümper (Bochum), Dieter Fürst (Bonn), Wolfgang Obermann (Bochum) and Ralph Knöll (Stockholm) for sharing antibodies. This work was supported by grants from the German Research Foundation (SFB1002, TPA08) and the IZKF Münster (Li1/ 029/20) to W.A.L. and the FoRUM Research Funding of the Medical Faculty, Ruhr University Bochum (F928R-2018) to S.S.

\section{Author contributions}

S.S. conceived the study approach and design, carried out most of the experiments, analyzed data, wrote a manuscript draft, and generated figures. A.U. performed EM and immuno-EM. Y.L. performed AFM-based nanoindentation and myofiber force measurements. A.V. and M.v.F.-S. performed PCRs, agarose and protein gel electrophoresis and western blotting. A.S., N.C., and L.L. sampled muscle biopsies from CIM patients. T.B. and L.L. provided advice on study design and made revisions to the manuscript. All authors commented on the manuscript. W.A.L. refined the study concept, analyzed data, finalized the manuscript, generated figures, and provided funding.

\section{Funding}

Open Access funding provided by Projekt DEAL.

\section{Competing interests}

The authors declare no competing interests.

\section{Additional information}

Supplementary information is available for this paper at https://doi.org/10.1038/s41467 020-18131-2.

Correspondence and requests for materials should be addressed to S.S. or W.A.L.

Peer review information Nature Communications thanks Lucas Smith and the other, anonymous, reviewer(s) for their contribution to the peer review of this work. Peer reviewer reports are available.

Reprints and permission information is available at http://www.nature.com/reprints

Publisher's note Springer Nature remains neutral with regard to jurisdictional claims in published maps and institutional affiliations. 
(c) (i) Open Access This article is licensed under a Creative Commons Attribution 4.0 International License, which permits use, sharing, adaptation, distribution and reproduction in any medium or format, as long as you give appropriate credit to the original author(s) and the source, provide a link to the Creative Commons license, and indicate if changes were made. The images or other third party material in this article are included in the article's Creative Commons license, unless indicated otherwise in a credit line to the material. If material is not included in the article's Creative Commons license and your intended use is not permitted by statutory regulation or exceeds the permitted use, you will need to obtain permission directly from the copyright holder. To view a copy of this license, visit http://creativecommons.org/ licenses/by/4.0/.

(C) The Author(s) 2020 